\title{
Advances in healthcare wearable devices
}

\author{
Sheikh M. A. Iqbal ${ }^{1,2}$, Imadeldin Mahgoub ${ }^{1}, E$ Du$^{3}$, Mary Ann Leavitt ${ }^{4}$ and Waseem Asghar $\mathbb{D}^{1,2,5 凶}$
}

Wearable devices have found numerous applications in healthcare ranging from physiological diseases, such as cardiovascular diseases, hypertension and muscle disorders to neurocognitive disorders, such as Parkinson's disease, Alzheimer's disease and other psychological diseases. Different types of wearables are used for this purpose, for example, skin-based wearables including tattoobased wearables, textile-based wearables, and biofluidic-based wearables. Recently, wearables have also shown encouraging improvements as a drug delivery system; therefore, enhancing its utility towards personalized healthcare. These wearables contain inherent challenges, which need to be addressed before their commercialization as a fully personalized healthcare system. This paper reviews different types of wearable devices currently being used in the healthcare field. It also highlights their efficacy in monitoring different diseases and applications of healthcare wearable devices (HWDs) for diagnostic and treatment purposes. Additionally, current challenges and limitations of these wearables in the field of healthcare along with their future perspectives are also reviewed.

npj Flexible Electronics (2021)5:9; https://doi.org/10.1038/s41528-021-00107-x

\section{INTRODUCTION}

Continuous and real-time monitoring is essential for better management of patients with chronic illnesses, including, cardiovascular diseases, diabetes, and neurological disorders. According to the World Health Organization (WHO), chronic diseases account for three quarters (75\%) of all deaths around the world and impose high economic burdens ${ }^{1}$. Therefore, different strategies are required for the monitoring and diagnosis of such diseases and an effective strategy in this regard is $\mathrm{HWDs}^{2}$. Wearable devices are defined as devices, which are worn on the human body or on clothing ${ }^{3}$. They consist of a target receptor and a transducer. A receptor recognizes the target analyte and responds accordingly ${ }^{4}$. The transducer then convert the receptor's response into a useful signal ${ }^{5}$. Several studies have reported applications of wearable devices in different fields; as a result, these devices have shown promising results in the field of healthcare due to their ability of deformability and compliance. These HWDs provide a better understanding of the changes inside a human body and can help in preventing and treating diseases.

Biosensors were initially used as invasive devices for controlled lab settings before their integration into wearable sensors. In 1956, Leland C. Clark, known as the "father of biosensors", was the first to use electrodes for the detection of the level of oxygen in blood $^{5}$. This device was meant for the continuous and real-time detection of oxygen in operating room settings during the cardiovascular surgery. The introduction of electrodes for healthcare purposes later led to the discovery of potentiometric biosensor for the detection of urea in 1969 by Guilbault and Montalvo, $\mathrm{Jr}^{5}$. The introduction of electrodes for healthcare lead to the commercialization of the first glucose analyzer in 1975 based upon Leland C. Clark's electrochemical biosensor ${ }^{6}$. With the miniaturization of electronics that leads to the micro and nanoelectronics and advancements in material sciences, integrated HWDs came into existence. These HWDs consists of electronic devices for the acquisition, processing and sharing of data. Conventional rigid and heavy electronic devices, for example, Printed Circuit Boards (PCBs) are not a suitable choice for HWDs therefore, significant advancements have been made in recent years in electronic devices in terms of their material, fabrication techniques, processing circuits and transceivers to enhance their compliance with HWDs. Recent advancements in HWD materials include biocompatible flexible materials for example, polyethylene naphthalate, polyethylene terephthalate (PET), Ecoflex, and Polydimethylsiloxane (PDMS), silicone-based materials, and thin film polymers for example, parylene ${ }^{2,7,8}$. These materials have high flexibility and stretchability, which makes them suitable substrates for HWDs. Ecoflex and PDMS have elongation limits of $900 \%$ and $400 \%$, respectively, where Ecoflex has Young's modulus close to human $\mathrm{skin}^{7}$. In comparison to conventional devices, these flexible electronic devices are not only low cost but are also power efficient in their consumption, which allow uninterrupted acquisition of data over long time. With emergence of communication modules like Bluetooth, Near Field Communication (NFC), Wi-Fi and Wireless body area networks (WBAN), visualization and sharing of the data in real time has become possible with HWDs ${ }^{9}$. These HWDs help in measuring different parameters and biopotentials. These biopotentials include electrocardiogram (ECG), which is a measure of heart performance, electromyogram (EMG) which is a measure of the activity of muscles in response to nerve stimulation, electroencephalogram (EEG), which is a measure of the performance of brain activity and electrooculogram (EOG), which records eye movements. These HWDs are extensively employed as noninvasive devices and particularly in point-of-care (POC) settings. Furthermore, the noninvasive nature of wearable devices has made therapeutic procedures simple and has decreased the risk of infection, which was previously related to blood ${ }^{10}$.

The POC wearable devices have revolutionized the healthcare system by decreasing the load on hospitals and by providing more reliable and timely information ${ }^{10,11}$. For this purpose, different types of wearable devices have been employed, e.g., epidermal-

\footnotetext{
${ }^{1}$ Department of Computer and Electrical Engineering \& Computer Science, Florida Atlantic University, Boca Raton, FL, USA. ${ }^{2}$ Asghar-Lab, Micro and Nanotechnology in Medicine, College of Engineering and Computer Science, Boca Raton, FL, USA. ${ }^{3}$ Department of Ocean and Mechanical Engineering, Florida Atlantic University, Boca Raton, FL, USA. ${ }^{4}$ Christine E. Lynn College of Nursing, Florida Atlantic University, Boca Raton, FL, USA. ${ }^{5}$ Department of Biological Sciences (Courtesy appointment), Florida Atlantic University, Boca Raton, FL, USA. ${ }^{\circledR}$ email: wasghar@fau.edu
} 


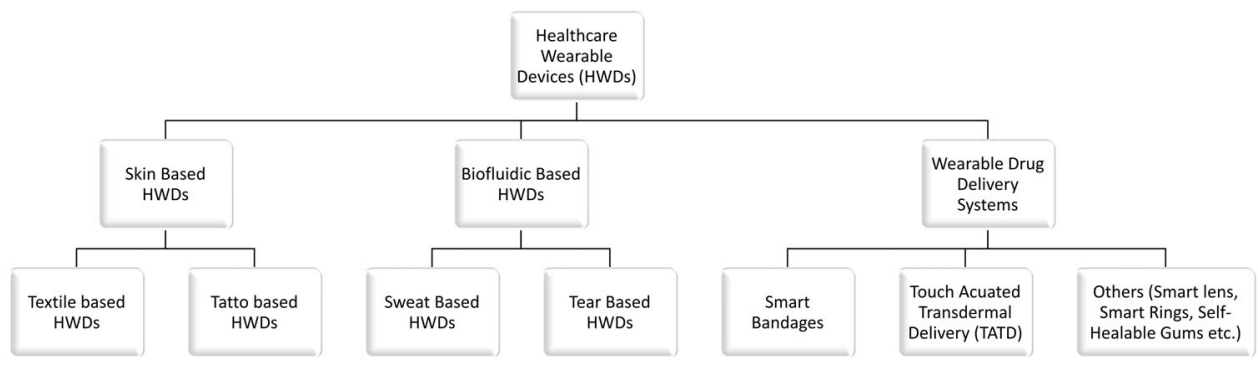

Fig. 1 Classification of Healthcare Wearable Devices (HWDs). Organization of the paper showing the classification of HWDs including Skin based HWDs, Biofluidic based HWDs, and Wearable Drug Delivery Systems along with their sub-categories.

based wearables, flexible wearables, and textile-based wearables $^{5,6}$. Wearables can be employed for different body parts, e.g., head-based wearables, eye-based wearables, and wrist-based wearables ${ }^{12}$. These wearables monitor different psychological and physiological parameters that can be used for the diagnoses of different diseases ${ }^{13}$. In fact, wearable devices can be integrated with different sampling platforms for sensing different chemical parameters from bodily fluids e.g., saliva, blood, urine, sweat, etc. $^{6,7}$. Additionally, these HWDs can be used for the delivery of drugs in a more controlled and efficient manner in comparison to traditional drug delivery systems ${ }^{14-16}$.

This paper reviews different types of wearable devices including different noninvasive wearables (including skin-based wearables), biofluidic-based wearables (including saliva, urine, and tears), and applications of wearables for drug delivery systems. Skin-based HWDs are further discussed as textile-based HWDs and tattoo-based HWDs as shown in Fig. 1. It highlights their efficacy in monitoring different diseases and applications of HWDs for the diagnostic and treatment purposes. The paper also compares some important commercially available HWDs. Additionally, current challenges and limitations of these wearables in the field of healthcare along with their future perspectives are also reviewed.

\section{SKIN-BASED WEARABLE DEVICES}

As skin covers most of the human body, it serves as an optimal mode for noninvasive healthcare wearable devices. Skin-based wearable devices can be used for physiological and psychological monitoring essential for the treatment of different diseases, for example cardiovascular and neuromuscular diseases. Additionally, it can also be used for the diagnosis of different diseases using qualitative and quantitative analysis of skin secretions, such as sweat. Based on the type of skin contact skin-based wearable devices can be either textile based or epidermal based. Textilebased wearable devices involve embedding essential sensors in clothes whereas epidermal-based wearable devices involve the direct attachment of wearables to the skin like a tattoo, generally known as electronic skin (e-skin). The following sections highlight the applications of textile-based HWDs and tattoo-based HWDs for the monitoring and diagnosing of different diseases.

\section{Textile-based HWDs}

Textiles have been around for centuries and are readily available. Traditionally, textiles and clothing have been perceived as keeping humans warm and for esthetics. Due to their accessibility and comfortability, they can be used for sensing important parameters, such as body temperature, heart rate, and respiration rate $^{17,18}$. Such HWDs are commonly known as electronic textiles or e-textiles. E-textiles are clothes embedded with sensors and conductive materials. The stretchable nature and large-scale skin contact make textiles an optimal medium for HWDs ${ }^{19}$. With the emergence of graphene, carbon nanotubes, and nanowires, a number of efforts have been made to incorporate sensors into clothing for continuous monitoring ${ }^{20-22}$. One such effort is by Yapici et al. who have developed an intelligent textile-based HWD for the monitoring of ECG ${ }^{23}$. Traditionally, ECG is monitored by using gel-based $\mathrm{Ag} / \mathrm{AgCl}$ electrode cables, which are not comfortable for the wearer. For textile-based ECG monitoring, graphene functionalized cloth has been embedded with ECG sensors ${ }^{23}$. Graphene has been used for this purpose because of its excellent material properties and high correlation with conventional gel-based ECG monitoring ${ }^{24}$. A comparison of the traditional $\mathrm{Ag} / \mathrm{AgCl}$ electrodes with the ECG HWD is shown in Fig. $2 \mathrm{a}^{23}$. It can be seen that graphene functionalized textile electrodes highly correlates with the conventional $\mathrm{Ag} / \mathrm{AgCl} \mathrm{ECG}$ electrodes with a maximum correlation of $97.0 \%{ }^{23}$. However, the graphene functionalized e-textile electrodes for ECG has higher electrode-skin impedance $(87.5-55 \mathrm{k} \Omega$ ) than the conventional $\mathrm{Ag} / \mathrm{AgCl}$ electrodes (50.9-20 k $\Omega$ ), which distorts the ECG and requires additional components like buffer amplifiers and adaptive filters ${ }^{23}$. Similarly, Arquilla et al. have tested textile electrodes for ECG monitoring using HWDs ${ }^{25}$. They have developed a chest-based ECG system by sewing ECG electrodes into the textile instead of using gel electrodes. This sensor system has been applied on eight different subjects (five males and three females) to validate the accuracy of textile-based ECG with the traditional gel-based ECG electrode. Differences between the heart rate and R-R intervals of ECGs from both systems were minimal and are shown in Fig. $2 b-d$. The statistical parameters for differences in heart rate are $t=-0.70$ and $p>0.5$ and $t=1.43$ and $p>0.1$ for $\mathrm{R}-\mathrm{R}$ interval and with $\mathrm{a}$ high correlation coefficient of $0.94^{25}$. However, this ECG sensor has not been tested during movement, which imposes considerable challenges in maintaining the skin-electrode contact and hence is mandatory for continuous ECG monitoring ${ }^{25}$.

Moreover, Wicaksono et al. have also developed an electronic textile comfortable suit (E-TeCS), as shown in Fig. $2 \mathrm{e}^{26}$. The E-TeCS provides temperature sensing of skin with a precision of $0.1^{\circ} \mathrm{C}$ as well as heart and respiration rates at a precision rate of $0.0012^{-2}$ using inertial sensing ${ }^{26}$. Additionally, washability and degradability tests were also conducted for the E-TeCS and high rigidity along with confirmation for no flakes or discoloration up to ten cycles of washing were observed, which makes it suitable for everyday use. Likewise, the use of HWDs can also be extended for other bipotential signals like EEG and EOG, which are required to be monitored for the treatment and diagnosis of different diseases ${ }^{27}$. Gao et al. have developed a multisensory textile-based HWD for the simultaneous detection of EEG from the forehead and sweat rate $^{28}$. This wearable utilizes silver $(\mathrm{Ag})$ as a conductive material embedded inside the textile electrode to record an EEG as shown in Fig. $2 \mathrm{f}^{28}$. The multisensor wearable consists of an EEG recording module and SHT20 chip for the relative humidity measurement. It has eight channels that allow independent recording of the EEG using different electrodes and the relative humidity is used to for the indirect measurement of the sweat rate. The multisensory HWD was compared with traditional Ag based wet electrodes 

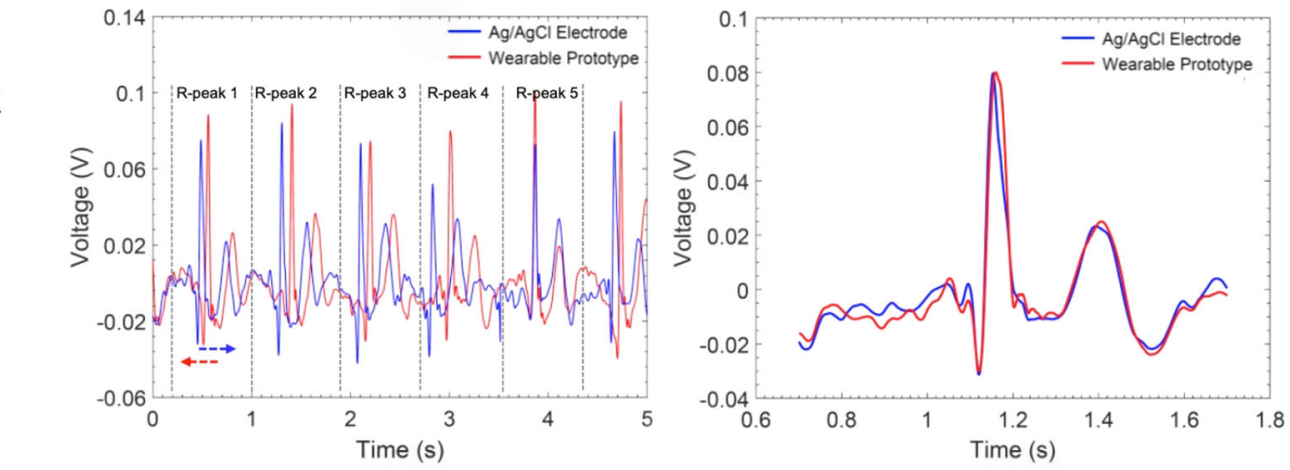

b

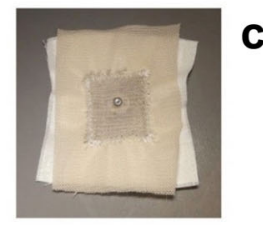

C

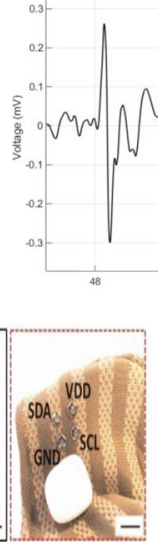

e

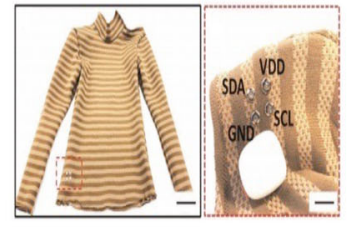

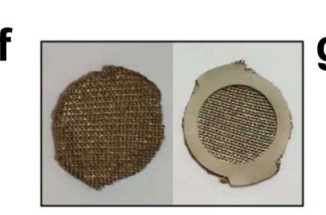

h

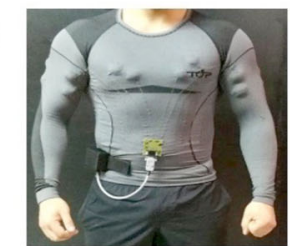

d

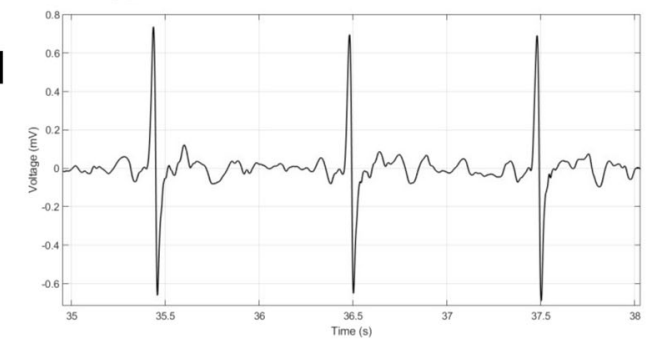

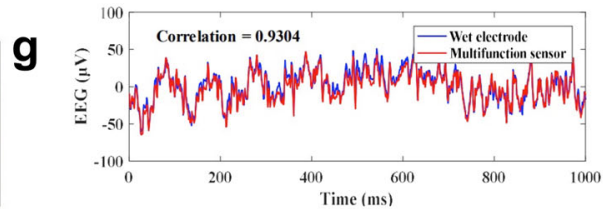

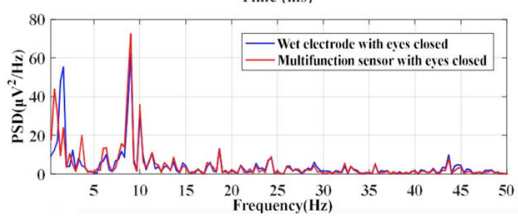

Fig. 2 Textile-based HWDs for measuring vital bio signals for example ECG, EEG, and EMG. a Comparison of ECG signals from Ag/AgCl electrodes and ECG HWD. Reproduced with permission from Yapici et al. ${ }^{23}$ Copyright $\odot$ Sensors, 2017. b Textile-based ECG electrode $\mathbf{c}$, d ECG vs time graphs of the textile-based ECG electrode. Reproduced with permission from Arquilla et al. ${ }^{25}$ Copyright $\odot$ Sensors, 2020 . e E-TeCS wearable with sensors on the shirt. Reproduced with permission from Wicaksono et al. ${ }^{26}$ Copyright $\odot$ npj Flexible Electronics, 2020. f Multisensor textile-based electrode for EEG and sweat rate measurement. $\mathbf{g}$ Comparison of textile-based electrode, as illustrated in $\mathbf{f}$, with traditional EEG electrode for alpha rhythm detection during eyes closed. Reproduced with permission from Gao et al. ${ }^{28}$ Copyright $\odot$ IEEE Sensors Journal, 2020. h Wearable shirt for the monitoring of EMG. Reproduced with permission from Pino et al. ${ }^{30}$ Copyright $\odot$ IEEE Conference of the IEEE Engineering in Medicine and Biology Society, 2018.

used for EEG measurement and was highly correlated. Figure $2 \mathrm{~g}$ shows results for alpha wave acquisition and their power spectral density (PSD) with eyes closed. Alpha rhythm is a standard electrical brain response with frequencies ranging from 8 to $15 \mathrm{~Hz}$ and usually more evident while eyes are closed. Correlation coefficients of $93.04 \%$ and $81.69 \%$ between the two electrodes have been found, in closed and opened eyes conditions, respectively. However, the skin-electrode impedance of the multisensory HWD decreases in the presence of sweat from $30-20$ to $6 \mathrm{k} \Omega$ in comparison to standard Ag wet electrodes that maintains small skin-electrode impedance irrespective of sweat ${ }^{28}$. Moreover, electrodes embedded inside the textile can also be used for EMG, which measures the electrical activity of muscles in response to nerve stimulation and is used for the detection of muscle or motor neuron abnormalities ${ }^{29}$. Pino et al. have proposed a wearable shirt to monitor EMG that provides essential feedback during exercise ${ }^{30}$. The wearable shirt is shown in the Fig. $2 \mathrm{~h}$ and consists of six dry electrodes embedded inside the shirt to measure EMG signals. These EMG signals are then sent to monitoring system using Bluetooth for signal processing.
The system sends an alert in case of a muscle fatigue, which is calculated using Dimitrov fatigue index $(\mathrm{FI})^{31}$.

Textile-based HWDs experience distortion in their results due to loose contact between skin and HWD. This requires additional signal processing techniques on raw data for acquiring clean signals. Moreover, textile-based HWDs provide comfortability along with real-time and continuous monitoring of the wearer, but their stability decreases with repeated washing due to the involvement of bio-recognizable molecules. The instability involved with textile-based HWDs may be partially resolved with tattoo-based HWDs.

\section{E-skin or tattoo-based HWDs}

Traditionally, tattoos have been perceived as a form of body art because of its pliability and compliance. These properties can be used for monitoring and diagnostic purposes. Currently, e-skins are widely used for the detection of electrical and physical parameters such as ECG, EEG, and EMG. Of these, the ECG is easiest to detect because of its high amplitude, of the order of 
a
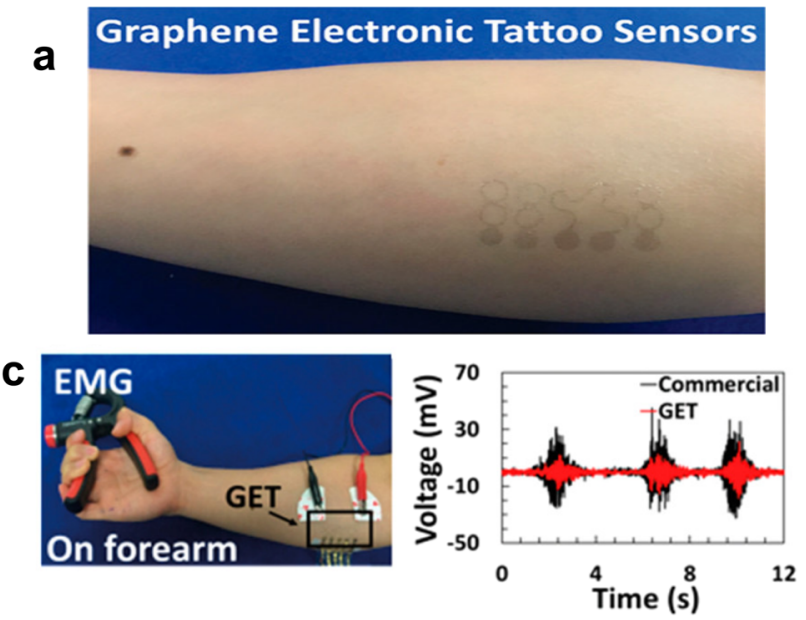

b
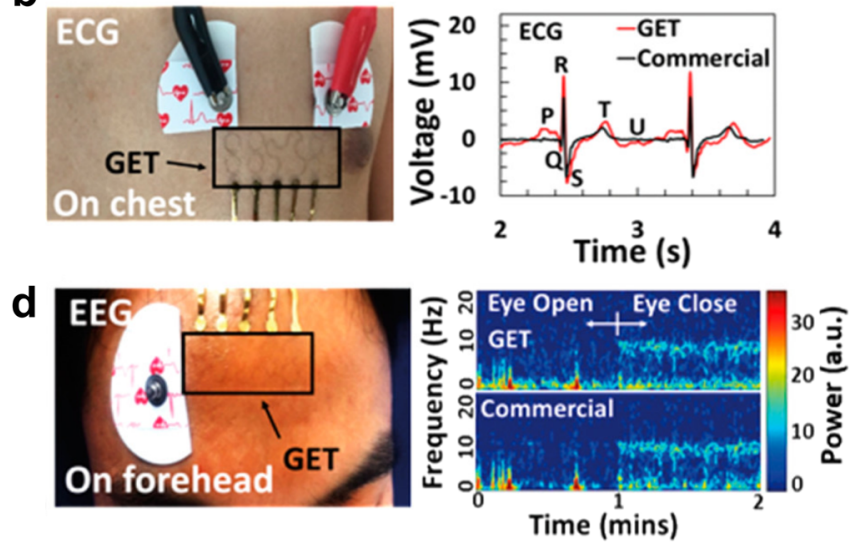

Fig. 3 E-skin-based HWDs. a A graphene electronic tattoo (GET) embossed on the forearm. b A comparison of ECG acquired using GET and commercial ECG monitor from chest. c EMG signals using GET from forearm. $\mathbf{d}$ EEG values using GET from forehead and frequency response of EEG during open and closed eyes condition highlighting the presence of alpha waves during eyes closed. Reproduced with permission from Ameri et al. ${ }^{33}$ Copyright $\odot$ ACS Nano, 2017.

$\sim 1 \mathrm{mV}$. This allows an accurate, noninvasive detection of heart signals through the $\mathrm{skin}^{32}$. In cardiovascular arrhythmias like tachycardia and bradycardia, ECG is the first point of reference for diagnosis and treatment. As mentioned, earlier, conventional ECG monitors require the attachment of gel-based electrode cables along with external electronic instrumentations for signal acquisition and can be uncomfortable for the wearer. Furthermore, these ECG monitors are mostly used in controlled lab or hospital settings and therefore cannot be carried with the wearer at all times. Many patients suffering from heart disease would benefit from continuous monitoring of their heart rhythm but a daily visit to a hospital poses an economic and scheduling burden. Moreover, tattoo-based ECG monitoring systems can resolve the instability and sensitivity issues of textile-based ECG monitors, due to their miniaturization and stretchability. They are also more flexible and comfortable for the wearer. A notable example of a tattoo-based HWDs ECG monitor is described by Ameri et al. and shown in Fig. $3 a^{33}$. It consists of miniaturized electronic components built on a graphene/polymethylmethacrylate (Gr/PMMA) bilayer substrate. The graphene electronic tattoo (GET) is fabricated using a wet transfer, dry patterning method and has $463 \pm 30 \mathrm{~nm}$ thickness with $\sim 85 \%$ optical transparency and more than $40 \%$ stretchability. The high stretchability and optical transparency make it light enough to be embossed on skin, like a tattoo. It binds to the skin using Van der Waals forces, which makes it mechanically invisible for the wearer. The GET is a comprehensive epidermal electronic system that is effective for different biopotentials like ECG, EMG, and EEG as shown in Fig. $3 a-d^{33}$. This HWD reports a clean ECG with high signal to noise ratio, comparable to conventional bulk gel-based electrodes. The module also works for other electrophysiological parameters, for example, EEG and EMG by embossing it on different parts of the body. Figure $3 a-d$ shows the acquisition of ECG from the chest, EMG from the forearm and detection of the presence of alpha rhythms in EEG from the forehead. Moreover, it has low skin impedance, comparable with Ag electrode, for $52 \mathrm{~h}$, however, $96 \mathrm{~h}$ after its application, crack starts to appear during the test, which increases its skin impedance, making it unsuitable for further use ${ }^{33}$.

Moreover, Dae et al. used a self-powered piezoelectric sensor for the continuous and real-time monitoring of the arterial pulse $^{34}$. The arterial pulse is a measure of heart's contraction rate, which is a component of cardiac output (heart rate $\times$ stroke volume $)^{34,35}$. A piezoelectric sensor converts the pressure created due to arterial pulses into electrical pulses. In-time detection of the abnormalities in atrial pulses could lead to the prevention of serious cardiac diseases. Monitoring arterial pressure may potentially help in the diagnosis of cardiac and blood diseases $^{36,37}$. This self-powered tattoo-based HWDs is POC in nature and mitigates the high-power consumption required by conventional arterial monitors. Moreover, data are shared wirelessly in case of any abnormality detected in arterial pressure signals using smartphone modality. It utilizes $\mathrm{BaTiO}_{3}$ and $\mathrm{Pb}\left[\mathrm{Zr}_{\mathrm{x}}, \mathrm{Ti}_{1-\mathrm{x}}\right] \mathrm{O}_{3}(\mathrm{PZT})$, active materials for the microelectromechanical sensors, due to their high piezoelectric coefficients. A thin layer of plastic was covered with PZT using laser lift-off technique (ILLO), which is an inorganic based technique for transferring polymers on surfaces ${ }^{38}$. The ultrathin layer of PZT adheres to the epidermis and responds to changes in arterial pulses. The self-powered PZT sensor was used for the detection of arterial pulses before and after the exercise. Before exercising, $V_{p p}$ of the arterial pulse is $81.5 \mathrm{mV}$ whereas after exercising its amplitude is $100 \mathrm{mV}$, an increase of $\sim 22 \%$ due to the increase in heart rate after the exercise. The same wearable was used for experiments in monitoring human respiratory activities, trachea movement and heart rhythm. These experiments have shown a sensitivity of $\approx 0.018 \mathrm{kPa}^{-1}$ and response time of $\approx 60 \mathrm{~ms}$ with excellent mechanical stability. However, the self-powered tattoo requires biocompatible piezoelectric material for clinical trials ${ }^{34}$.

Furthermore, it is estimated that over one billion people worldwide have hypertension, or elevated blood pressure (BP). Out of 1 billion, two-thirds are in developing countries who also lack adequate healthcare facilities ${ }^{39}$. Daily monitoring of BP is essential for these patients as hypertension is often asymptomatic. Due to the lack of monitoring, hypertension is one of the major causes of premature death across the world ${ }^{39}$. Traditionally, BP monitors are based on auscultative techniques with an inflatable cuff and on first and fifth Korotkoff sounds ${ }^{40}$. This measures systolic blood pressure (SBP), a number that represents the pressure in arteries when the heart muscle contracts, and diastolic blood pressure (DBP), a number that represents the pressure when the heart muscle relaxes ${ }^{40}$. Accurate $\mathrm{BP}$ measurement requires trained technicians, which adds to the challenge to meet the WHO target to reduce hypertension by $25 \%$ by 2025 . However, HWDs can provide a suitable wearable for the POC setting to help achieve the aforementioned target. One such wearable is described by Luo et al. who has developed a cuff-less BP measuring device for real time, continuous measurement of blood pressure using tattoo-based $\mathrm{HWD}^{41}$. The HWD integrates a thin 

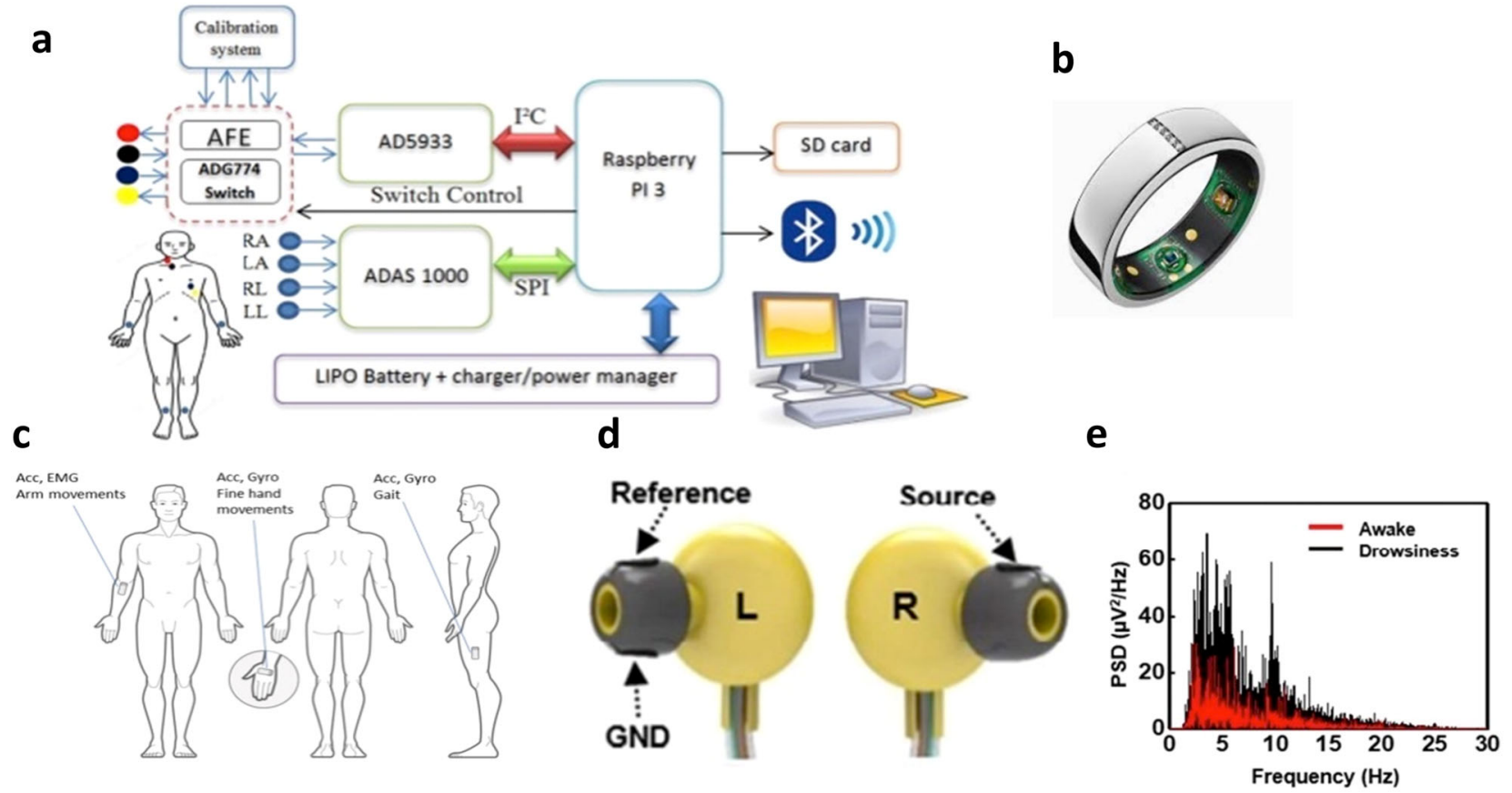

Fig. 4 Other competitive skin-based HWDs. a Schematic of the Heart Failure Prediction System. Reproduced with permission from Hafid et al. ${ }^{48}$ Copyright $\odot$ IEEE Journal Of Biomedical and Health Informatics,2018. b Oura Ring with miniaturized sensors on it to diagnose different illnesses. Reproduced with permission ${ }^{50}$ Copyright (C) Oura Ring, 2020. c Position of sensors for the detection of bradykinesia and tremor features of PD. Reproduced with permission from Lonini et al. ${ }^{71}$ Copyright @ npj Digital Medicine, 2018. d Earphones for the portable acquisition of EEG with reference and ground electrodes on left earphone and source electrode on right earphone. e Power Spectral Density (PSD) for the differentiation of the state of drowsiness and awake state using EEG from HWD as illustrated in d. Reproduced with permission from Lee et al. ${ }^{78}$ Copyright $\odot$ ACS Applied Materials and Interfaces, 2018.

flexible piezoelectric sensor (FPS) with epidermal ECG sensor for the cuff-less measurement of $\mathrm{BP}$, which is much more comfortable than its conventional counterpart. The patch uses beat-to-beat BP measurements from ECG along with a parametric model, which is very sensitive to minute changes in physiological signals in the epidermis. The epidermal signals and ECG signals were obtained using the piezoelectric patch along with SBP and DBP. Average BP values for conventional cuff-based BP monitor and cuff-less BP HWD were evaluated, and they were found to be comparable. The conventional cuff-based BP monitor showed $\mathrm{SBP}=123.8 \pm$ $5.7 \mathrm{mmHg}$ and DBP $=86.8 \pm 4.4 \mathrm{mmHg}$, while cuff-less BP HWD showed $\mathrm{SBP}=130.3 \pm 0.5 \mathrm{mmHg}$ and $\mathrm{DBP}=86.5 \pm 0.5 \mathrm{mmHg}$. These results indicate that cuff-less $B P$ wearable provides high correlation with a conventional BP monitor for both SBP and DBP values. Additionally, the patch utilizes only $3 \mathrm{nW}$ of power, in comparison to photoplethysmogram (PPG) based devices that consumes tens of milliwatts of power, making it more efficient and optimal for home-based settings in low and middle-income countries $^{41-43}$.

\section{Other competitive skin-based HWDs including wearable vests,} smart rings, and earphones

There are other skin-based biosensors, which are not textile or tattoo based. Various wrist bands, such as smartwatches, wearable vests, skin patches, and implantable HWDs, use different monitoring biomarkers. Schreiner et al. and Leonard et al. have monitored respiratory rate, an essential parameter for many respiratory and cardiovascular diseases by using alternative wearable technologies ${ }^{35,44}$. Schreiner et al. proposed a chestbased wearable to monitor respiratory rate using pulse oximetry ${ }^{44}$. Similarly, heart failure is another clinical syndrome, which requires continuous monitoring. A structural abnormality, which hinders heart from not pumping sufficient blood to fulfill the needs of the body, heart failure affects about 26 million people globally ${ }^{45}$. Heart failure requires real time, continuous monitoring, which increases the economic burden; HWDs can facilitate the daily monitoring of such patients. A number of HWDs have been reported that go beyond diagnosing or monitoring heart failure but can predict it days before it even occurs ${ }^{31,44,46-48}$. Hafid et al. proposed a wearable system to predict heart failure using multiple parameters, such as thoracic impedance, heart rate, $E C G$, and blood oxygen level ${ }^{48}$. All of these parameters are measured noninvasively using electronic circuitry, a schematic of which is shown in Fig. 4a. The most important parameter may be the thoracic impedance, which is the measure of the resistance to the flow of ions due to the retention of fluid in the thoracic region of the human body ${ }^{49}$. Days before heart failure symptoms such as shortness of breath are noted by the patient, fluid is retained in the lungs, which decreases thoracic impedance ${ }^{48}$. The wearable system tracks the thoracic impedance and other parameters including heart rate, ECG and level of oxygen in the blood. If the respective values exceed the safe threshold, the HWD generates an alert using wireless transmission over a cloud server to the wearer and to the medical provider. The clinician can then make necessary interventions. However, the prognosis module has inefficient power consumption taking it $8 \mathrm{~h}$ to charge and can only measure continuously for $5 \mathrm{~h}$, which limits its utility as a HWD for continuous monitoring at all times ${ }^{48}$. Similarly, another HWD, Oura Ring monitors key physiological parameters to help the wearer have a restful sleep ${ }^{50}$. Oura Ring is a metallic ring, as shown in Fig. 4b, with miniaturized sensors to monitor physiological parameters, such as heart rate, body temperature, and breathing ${ }^{51}$. These parameters can be monitored for illnesses like cold, flu, and even the novel Coronavirus 2019 (SARS-CoV-2). Severe acute respiratory syndrome (SARS) outbroke in 2003 and the Middle East respiratory syndrome (MERS) in 2012; SARS-CoV-2 is the third and the most recent of coronaviruses ${ }^{52,53}$. SARS-CoV-2 has currently become a global pandemic with 66 million cases 
and 1.54 million deaths worldwide ${ }^{54}$. Oura Ring utilizes the aforementioned parameters to potentially detect symptoms of SARS-CoV-2 in home settings. The wearable ring detects common SARS-CoV-2 symptoms such as fever, cough, fatigue, and difficulty breathing with $90 \%$ accuracy ${ }^{55}$. Moreover, the ring has a battery life of 4-7 days, depending upon the usage, and takes only 20-80 min to recharge ${ }^{56}$.

There are also HWDs for use in the oral cavity. Kim et al. have developed a mouthguard for the monitoring of saliva uric acid ${ }^{57}$. Similarly, Mannoor et al. have developed an oral cavity HWD for the detection of bacteria on tooth enamel ${ }^{58}$. The HWD is based on graphene because of its high strength $\left(42 \mathrm{Nm}^{-1}\right)$ and Young's Modulus $(\sim 1 \mathrm{TPa})^{59}$. Antimicrobial peptides (AMPs) were probed on graphene for the detection of bacteria at a single cell level. Moreover, the resonant coil excludes the need of an external power source making it miniaturized and power efficient. Results have been reported for the Escherichia coli (E. coli) bacterium, a foodborne infection. E. coli is commonly found in the intestines of humans and warm blooded animals ${ }^{60}$. If food is contaminated with E. coli, it can cause severe diseases such as gastroenteritis (food poisoning) ${ }^{61}$. The graphene-based nanosensor is attached to the tooth and can remotely monitor respiration and bacteria from the saliva based upon the change in its resistance ${ }^{58}$. Odorranin-HP AMP has been probed for the detection of E. coli because of its strong activity towards this organism. As soon as E. coli binds with the AMP immobilized graphene, the resistance of graphene decreases and helps in detecting E. coli with a limit of detection of 1 bacterium $\mu \mathrm{L}^{[-158}$. Results are justified with fluorescent images for the presence of E. coli. Similar experiments were performed for the detection of helicobacter pylori $(H$. pylori), a gram-negative bacterium usually found in saliva, and stomach. $\mathrm{H}$. pylori is one of the leading cause for over $90 \%$ of stomach cancers and duodenal ulcers $^{62,63}$. The lower limit for the detection of $\mathrm{H}$. pylori is $\sim 100 \mathrm{H}$. pylori cells using tooth enamel HWD and shows linear relation with its logarithmic concentration.

Furthermore, neurological diseases can also be managed efficiently with continuous monitoring. EEG, EOG, and motion monitoring can be used to diagnose and keep track of severe neurological diseases, like epilepsy, Parkinson's disease (PD) and Alzheimer's disease (AD). HWDs like ocular wearables can be used for the indirect detection of neurological diseases using $E \mathrm{GG}^{64}$. Wearable sensors have been used for the monitoring of patients with $\mathrm{PD}^{65,66}$. PD is a neurological disease that involves muscular rigidity and marked by bradykinesia or slow movement ${ }^{67}$. It is the second most common degenerative disease after Alzheimer's, and it is estimated that 10 million people around the world are affected with $P D^{68,69}$. Lima et al. have used body fall sensors for quantifying the probability of falling in PD patients in homebased settings. The study shows that the ratio of falling nearly doubles in PD patients in comparison to healthy individuals ${ }^{70}$. Similarly, Lonini et al. have also developed an intelligent system based on machine learning algorithms for the detection of PD symptoms like bradykinesia and tremor (involuntary movements) ${ }^{71,72}$. Different motion sensors like, accelerometers, gyrometers, electromyography, and inertial sensors, are used as shown in Fig. $4 \mathrm{c}^{71}$. For the detection of aforementioned symptoms, subjects performed different tasks like, typing and walking, over a course of time. Using machine learning algorithms based on statistical ensembles along with convolutional neural networks the clinician could detect bradykinesia and tremor. However, the study was conducted on a limited number of participants (19) and therefore was unable to infer generalized conclusions about the detection of dyskinesia (impaired voluntary movement), another significant symptom of $\mathrm{PD}^{71,73}$. Similarly, dementia is another neurological disease, which is characterized by memory loss and difficulty in solving problems. There are 50 million cases worldwide and every year there are 10 million new cases of dementia ${ }^{74,75}$. Alzheimer's disease is the most common form of dementia. Almost $60-70 \%$ of dementia patients are diagnosed with Alzheimer's disease ${ }^{74}$. Monitoring of significant moments can help to support the care of dementia patients and HWDs can be employed for this purpose. Kwan et al. proposed a wearable, based on intelligent assistive technology (IAT) where IAT is an adaptive and assistive technology, integrated with advanced environment techniques of artificial intelligence $(\mathrm{Al})^{76}$. The wearable is tailored to an individual's physiological responses ${ }^{77}$. These physiological responses consist of heart rate, electrodermal activity-responsible for the emotional arousal and skin temperature. These signals are extracted using the autonomic nervous system (ANS) and using a triple point sensor (TPS), which can be worn on the fingertips using a Velcro strap. The IAT detects significant emotional events based upon their physiological response and provides feedback to dementia patients. Considering the importance of EEG in daily routine, portable, and flexible earphones have been developed by Lee et al. for the acquisition of $\mathrm{EEG}^{78}$. The earphones consist of three electrodes (Reference, Source and Ground) made up of a mixture of carbon nanotubes (CNTs), polydimethylsiloxane (PDMS) and silver nanowires (AgNW), as shown in Fig. $4 d^{78}$. The earphones are incapsulated in a plastic frame containing electronics, for example, conductive elastomers, signal transducers, and metal strip with soft earbuds for the convenient recording of EEG along with listening music at the same time. The earphones successfully differentiated the state of drowsiness from the awake state using the three-electrode system and results were communicated to a smartphone using a Bluetooth circuit as shown in Fig. 4e. However, as the earphones measure the EEG in crosstalk with music, EEG signals have low signal to noise ratio (SNR) and therefore requires additional amplifiers with post signal processing techniques for signal enhancement ${ }^{78}$.

\section{BIOFLUIDIC-BASED HWDS}

Body secretions like sweat, saliva, tears, and urine contain important biomarkers that are essential for monitoring and diagnostic purposes. HWDs can be used directly or through their integration with other platforms, for example, microfluidic platforms can be integrated for the extraction of useful information from different biofluids ${ }^{79}$. Microfluidic platforms of different materials can be used in HWDs for example, polymer-based microfluidic devices, paper-based microfluidic devices, and microsized needles known as microneedles ${ }^{80,81}$. The following sections highlight recent efforts in biofluidic-based HWDs classified according to the type of biofluids.

\section{Sweat-based HWDs}

Epidermal biofluids like sweat is an important indicator of changes taking place inside human body and hence can serve as an important parameter for the chemical and biological sensing. Sweat is composed of different biomarkers, for example, metabolites (e.g., glucose, lactate, urea etc.), proteins, nucleotides, and electrolytes such as chlorine, sodium etc. which have important diagnostic implications ${ }^{32}$. Sweat is readily available for chemical sensing and is distributed across the skin with more than 100 glands $\mathrm{cm}^{-2} 32$. Therefore, sweat can be used for the extraction of different chemical and biological parameters using HWDs to provide monitoring and diagnostics in POC settings ${ }^{82}$. Koh et al. have developed a flexible, soft, and stretchable device, based on microfluidics, for the colorimetric sensing of sweat biomarkers as shown in Fig. $5 a^{83}$. This HWD is capable of quantifying electrolytes, like chloride and hydronium ions, that are essential for monitoring in cystic fibrosis, a chronic inherited disease that affects the respiratory and digestive systems, by forming a thick mucus that can block lungs and can obstruct the pancreas ${ }^{84}$. People with cystic fibrosis tend to have a shorter life 
a

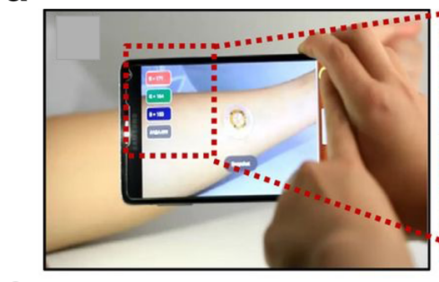

C

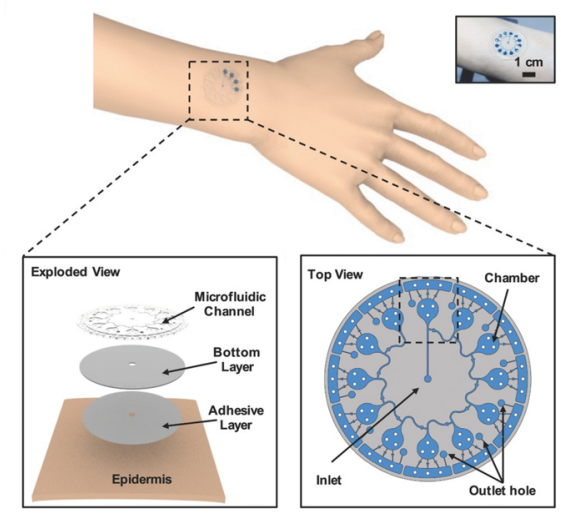

b

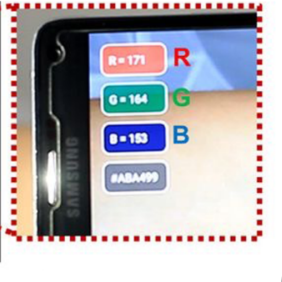

d
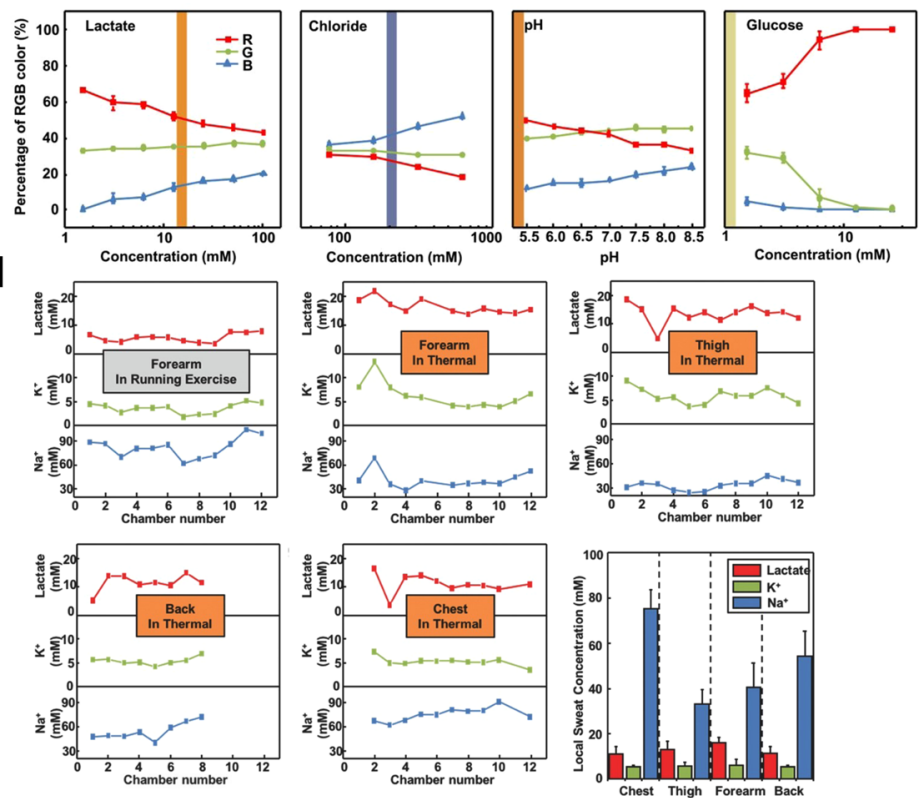

e

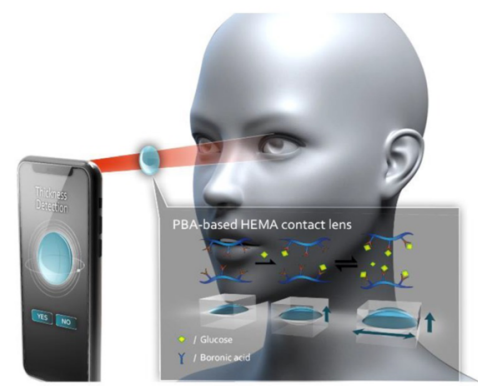

Fig. 5 Biofluidic-based HWDs. a Colorimetric analysis of sweat from the image captured using smartphone. b Quantification of the concentration of chloride, hydronium ions, and glucose and lactate levels using HWD as illustrated in a. Reproduced with permission from Koh et al. ${ }^{83}$ Copyright $\odot$ Science Translational Medicine, 2016. c A skin-like soft wearable microfluidic platform for the detection of different analytes from sweat. d Concentrations of sodium, potassium, and lactate at different body locations and at different chambers of the HWD illustrated in c. Reproduced with permission from Choi et al. ${ }^{90}$ Copyright $\odot$ Advanced Healthcare Materials, 2017. e Smart contact lens for the monitoring of diabetes using smartphone. Reproduced with permission from Lin et al. ${ }^{98}$ Copyright @ Sensors, 2018.

span than healthy individuals ${ }^{85}$. The microfluidic HWD can also provide quantification of glucose and lactate levels, which are essential for diabetic patients along with the loss of $\mathrm{pH}$, sweat rate, and total sweat loss. When the perspiration starts, the sweat is transferred into microfluidic reservoirs, where chromogenic reagents, like a mixture of glucose oxide and horseradish peroxide (HRP) for glucose level, respond to aforementioned biomarkers. Images of the change in color of the chemical analytes can be captured using a smartphone for the quantification of the concentration of chloride, hydronium ions, glucose, and lactate levels, as shown in Fig. $5 b^{83,86,87}$. The HWD continuously measures the concentrations of aforementioned electrolytes in excellent agreement with the laboratory analysis of the sweat. They have demonstrated the flexible microfluidic HWD for human studies on the fitness cycle in a controlled environment and for long distance bicycle racing in outdoor settings, to justify the absence of the leakage of fluid and any discomfort due to the microfluidic HWD during real life arid conditions. The microfluidic device is fabricated using the soft lithographic technique with PDMS as a material $^{83}$. Soft lithography offers high resolution, of the order of submicrometers, and due to its simplicity and flexibility it has been a widely used technique for fabricating biosensors $83,88,89$. However, it is a labor intensive and manually operated process ${ }^{88^{\circ}}$.
A similar soft microfluidic based HWD was developed by Choi et al. that uses microreservoirs for the detection of sodium, potassium, and lactate concentrations from sweat along with their temporal variations ${ }^{90}$. This wearable is a skin-like soft microfluidic platform that takes $1.8 \mu \mathrm{L}$ volume of sweat from an area $0.03 \mathrm{~cm}^{2}$ of skin in $0.8 \mathrm{~min}$ of sweating at $0.60 \mu \mathrm{L} \mathrm{min}{ }^{-1}$ rate as shown in Fig. 5c. The Fig. 5d shows different concentrations of sodium, potassium, and lactate at different body locations and at different chambers of the wearable. Concentrations of these analytes show variations at different times and at different spatial positions on the body and propose optimal spatial positions for the attachment of the wearable for sweat analysis. Paper-based microfluidic devices, commonly known as microfluidic paper-based analytical devices ( $\mu$ PADs), are another promising technique for fabricating biofluidic-based HWDs. $\mu$ PADs are biocompatible, low cost and offer high capillary action ${ }^{91,92}$. The multisensing patch for sweat monitoring by Anastasova et al. is a $\mu \mathrm{PADs}^{91}$. The patch consists of microneedles of $50 \mu \mathrm{m}$ diameter and measures the concentration of lactate, $\mathrm{pH}$ using sodium ions and temperature using amperometric sensing. The HWD demonstrates sensitivity of $71.90 \pm 0.8 \mathrm{mV}_{\text {unit }}{ }^{-1}$ and $56 \pm 1 \mathrm{mV}$ unit $^{-1}$ for $\mathrm{pH}$ and sodium ions respectively with a response time of around $\sim 90 \mathrm{~s}$. The wearable transmits data wirelessly for real-time monitoring of sweat in home-based settings. Moreover, the HWD ensures user 
compliance with overall thickness of as low as $180 \mu \mathrm{m}$ with double sided adhesion to ensure firm adhesion of the patch to skin. Another notable $\mu$ PADs is a Smart Wearable Sweat Patch (SWSP) sensor $^{92}$. SWSP consists of paper substrates, fluorescent sensing probes and microchannels ${ }^{92}$. The microchannels are made up of cotton to absorb sweat from the skin and to transport them to sensing probes to quantify analytes. The HWD measures the concentrations of glucose, lactate, and chloride ions from the sweat by quantifying the intensity of the fluorescent from each analyte, using a smartphone ${ }^{92}$. The device offers excellent statistical results for the identification of aforementioned analytes from sweat with limit of detection of $7 \mu \mathrm{m}, 0.4 \mathrm{mM}$, and $5 \mathrm{mM}$ and correlate coefficients of $0.990,0.988$, and 0.994 for glucose, lactate, and chloride ions, respectively. The device is low cost $(\sim 0.3)$ but cannot be used to measure concentrations of aforementioned analytes in real time ${ }^{92}$.

\section{Tears-based HWDs}

Tears are another important biofluid for the purpose of diagnosis and monitoring of different diseases. One such disease is diabetes and a number of HWDs have been developed for its diagnosis. Diabetes is a chronic metabolic diseases, with elevated levels of glucose or blood sugar, which can lead to serious damage to the heart, eye, kidneys, nerve, and blood vessels ${ }^{93}$. Globally, patients with diabetes have increased from 108 million to 463 million in 2020 and if necessary interventions are not taken then it is expected to increase, especially in middle and low-income countries $^{93,94}$. Prevention of the complications of diabetes, as proposed by WHO, includes a healthy lifestyle with continuous monitoring of glucose levels. Traditional portable glucometers were developed to measure the concentration of glucose in blood. However, it involves finger sticks that is uncomfortable for the patient and can lead to infections related to blood pathogens. Urine can also be used for blood glucose measurement, however, it is difficult to handle and can impose limitations when used at home-based settings. HWDs provide a convenient and comfortable wearable for measuring the blood sugar and instead of blood and urine, tears can be used for the glucose measurement. Sen et al. measured the concentration of glucose in tear samples and related it to the concentration of glucose from blood samples, which justified the efficacy of tears for glucose measurement ${ }^{95}$. It was shown that levels of glucose were much higher in tears for a diabetic patient as compared to blood and urine samples in healthy individuals. Therefore, several other HWDs have been developed in the past for the measurement of glucose using tears. Notable examples are commercialized products, like Triggerfish that monitors the intraocular pressure of glaucoma patients and Google lens for the diagnosis of diabetes by Google in collaboration with Novartis ${ }^{96,97}$. Lin et al. have developed a smart contact lens for the diagnosis and continuous monitoring of diabetes using tears ${ }^{98}$. The lens is made up of phenylboronic acid (PBA), a non-enzyme, and Hydroxyethyl methacrylate (HEMA), a monomer. It utilizes the reversible covalent interaction of the PBAHEMA-based contact lens with glucose for the monitoring of diabetes. The PBA-HEMA based contact lens swells in the presence of glucose and thereby increases its thickness. The contact lens thickness increases simultaneously with the increase in glucose level, at a linear rate, as shown in Fig. $5 \mathrm{e}^{98}$. The HWD has shown an excellent agreement in sensing the glucose ${ }^{98}$. It can be seen that the thickness of the lens increases linearly as the concentration of glucose increases from 0 to $20 \mathrm{mM}$ within $15 \mathrm{~min}$. The contact lens does not require embedded power circuits and additional photosensors; however, it only utilizes a smartphone for the detection of the change in thickness of lens. The smartphone captures the light reflected by the light-emitting diode on the smart lens. Images captured are then analyzed using an inbuilt software for the detection of glucose levels, as shown in Fig. $5 \mathrm{e}^{98}$.
The noninvasive nature of the lens and integration with a smartphone, makes it a suitable sensor for monitoring diabetes at home settings. The lens is lightweight, flexible, and transparent to ensure the patient's vision without obstruction. Moreover, integration with smartphone ensures the portability of the HWD to enhance the patient's compliance with HWD ${ }^{98}$.

As discussed, microfluidic based devices offer low cost and suitable platforms for biofluidic-based HWDs; however, they have inherent limitations ${ }^{99,100}$. These limitations include fluid leakage, contamination, and blockage due to debris and limited flexible materials make them inconvenient for the wearer ${ }^{99}$. However, with more flexible materials along with adequate flow rate optimization, these challenges can be overcome to fabricate lowcost microfluidics based HWDs.

\section{WEARABLE DRUG DELIVERY SYSTEMS}

As previously discussed, biodegradable and biocompatible materials are available due to advancements in materials. Such materials can be used for drug delivery systems, which can deliver drugs in a controlled manner ${ }^{101,102}$. One application of drug delivery, is bimatoprost, using an ocular ring made up of polypropylene for the treatment of glaucoma ${ }^{101}$. Glaucoma is an eye condition that damages the optic nerve and is the leading cause of blindness worldwide ${ }^{103}$. Bimatoprost is a medication generally used for the treatment of glaucoma, but fluctuations in its usage can minimize its efficacy. Therefore, the ocular ring, which is covered with silicone-bimatoprost matrix, slowly releases bimatoprost in a controlled manner, as shown in Fig. 6a. Similarly, Al-Shahbazi et al. have developed a self-healable and moldable gum for the personalized drug delivery ${ }^{104}$. The gum is made up of two materials, polyvinyl alcohol (PVA) and tannic acid (TA), collectively referred to as PATA, which belongs to a human friendly group of materials and makes it a bioactive material. The polymeric gum developed, as shown in the Fig. $6 \mathrm{~b}$, has high strength, stretchability, flexibility, toughness, and self-healing properties. The gum has been found to display antibacterial and anti-inflammatory properties and is flexible enough to be molded into any shape suitable for using it in wearables.

Similarly, Kim et al. have developed a touch actuated transdermal delivery (TATD) wearable for controlled drug delivery ${ }^{105}$. The TATD provides quantitative permeation control of the drug delivery in accordance with the force exerted by the touch on a wearable patch. The HWD consists of drug reservoirs that are refillable in nature, strain sensors for force detection, and microneedles for drug release as shown in Fig. $6 \mathrm{~d}^{105}$. With mathematical models between the force exerted and the quantity of drug released, a controlled drug delivery has been observed, which enables a real-time drug regulation. Similarly, Di et al. have also developed a mechanical force based drug delivery system ${ }^{106}$. This HWD consists of a stretchable elastomer with drug reservoirs in the form of polymeric nanoparticles loaded with drugs. These nanoparticles can be loaded with antibacterial drugs or antiinflammatory drugs. Likewise, the HWD produces encouraging results with anticancer therapy. The drug is released in a controlled manner by applying tensile strain on the elastomer and the rate of release of drug is per Poisson's ratio. The HWD is a skin mountable device and can be administered by the motion of muscles or joints, tendons or by external force using hands.

Moreover, T. Ly et al. have utilized the Bluetooth technology for the controlled release of insulin ${ }^{107}$. The Bluetooth enabled insulin pump is tubeless in nature and can hold up to 200 units of U-100 insulin. It consists of cannula insertion and automated priming along with a personalized diabetes manager, portable for the remote control of insulin delivery. In another report, Keum et al. have developed a smart contact lens for the detection of glucose levels from tears and for delivering drug for diabetic retinopathy therapy ${ }^{97}$. The contact lens is $14 \mathrm{~mm}$ in diameter, $200 \mu \mathrm{m}$ in 


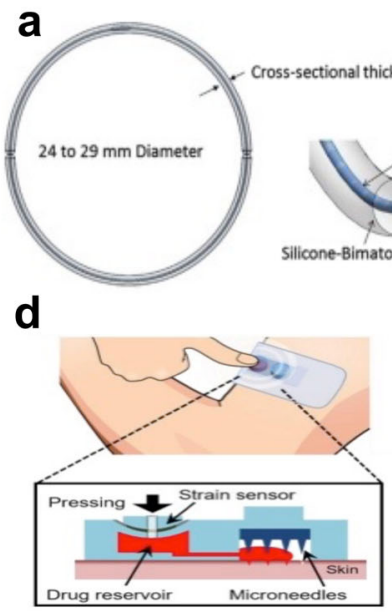

b

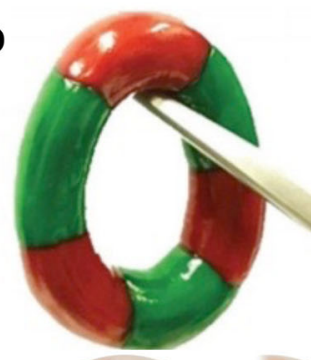

e
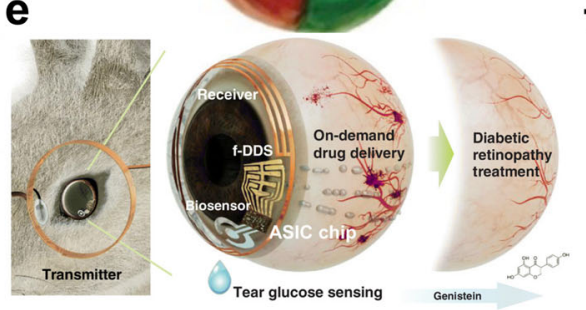

g

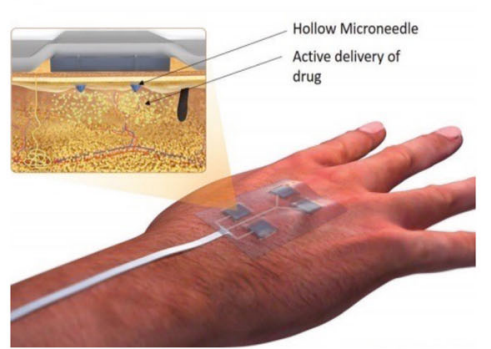

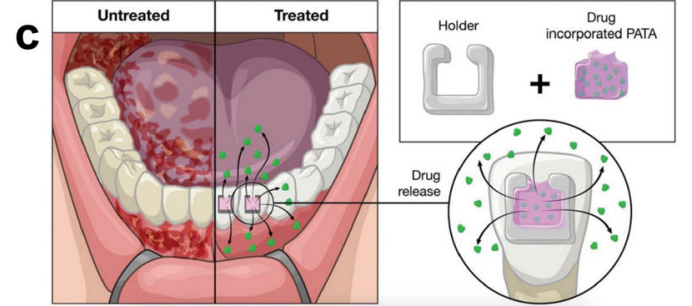

$\mathbf{f}$

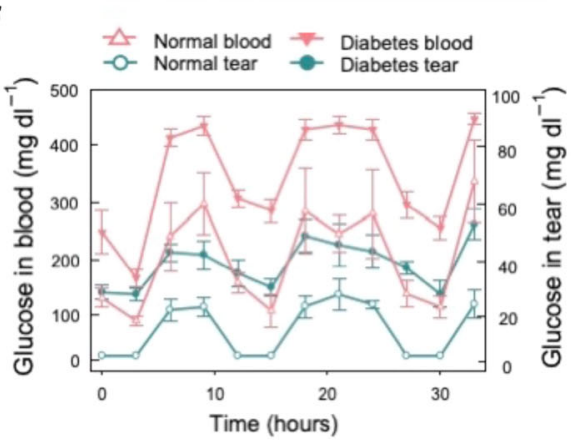

Fig. 6 HWDs for drug delivery. a Ocular Ring for the treatment of glaucoma. Reproduced with permission from Brandt et al. ${ }^{101}$ Copyright $\odot$ American Academy of Ophthalmology, 2017. b Self-healable gum made from PATA biomaterial. c Gum, as illustrated in b, filled with a drug and treated in an oral cavity. Reproduced with permission from Al-Shahbazi et al. ${ }^{104}$ Copyright $\odot$ Journal of Materials Chemistry B, 2020. d Working principle of touch actuated transdermal drug delivery. Reproduced with permission from Kim et al. ${ }^{105}$ Copyright $\odot$ Sensors and Actuators, 2018. e Smart contact lens for diabetic retinopathy therapy by detecting glucose level. $\mathbf{f}$ comparison of glucose levels in blood and tear for normal and diabatic person. Reproduced with permission from Keum et al. ${ }^{97}$ Copyright $\odot$, Science Advances, 2020. g Working principle of wirelessly controlled smart bandage. Reproduced with permission from Derakhshandeh et al. ${ }^{108}$ Copyright $\odot$, Advanced Functional Materials, 2020.

thickness with $8.0 \mathrm{~mm}$ radius curvature is based upon an ultrathin, biocompatible polymer and contains miniaturized electrical circuits along with a microcontroller chip for the continuous monitoring of glucose level from a tear. The contact lens as shown in the Fig. $6 \mathrm{e}$, consists of an electrochemical biosensor, a drug delivery system, a resonant inductive coupling to copper for wireless transfer of power from an external transmitter coil, an integrated microcontroller chip and a radio frequency (RF) system for communication. The biosensor contains three electrodes: Reference Electrode (RE), Working Electrode (WE), and Counter Electrode (CE) for the detection of glucose. Results in Fig. $6 \mathrm{f}$ show a comparison of glucose levels in blood and tears between healthy and diabetic individuals and it can be seen that at all times the difference in the level of glucose in both diabetic and healthy individuals, for both tears and blood, are high enough to diagnose diabetes from tears. Moreover, the contact lens has an integrated drug delivery system that allows an on-demand, controlled delivery of an antidiabetic drug. The delivery of the drug is controlled by on/off control of voltage. Drug delivery is electrically controlled using a gold (Au) membrane. Drug reservoirs are covered with Au anode electrode, a thin layer of Au membrane, which dissolves in phosphate buffer solution (PBS) in $40 \mathrm{~s}$. When a voltage of $1.8 \mathrm{~V}$ is applied, the drug is delivered, and results are then shared by the wireless transmission using an RF module, which makes it a suitable HWD at POC. Furthermore, Derakhshandeh et al. have proposed a smart bandage for the treatment of chronic wounds ${ }^{108}$. The bandage consists of microneedles, as shown in the Fig. $6 \mathrm{~g}$, for the delivery of pharmacological agents and vascular endothelial growth factors (VEGF) on the targeted wound. The bandage delivers the drug in a controlled and wireless manner. Moreover, the microneedles were fabricated using a 3D printer on polymeric substrate and microchannels were fabricated using PDMS. The smart bandage was experimented by delivering VEGF to chronic dermal wounds of diabetic mice and showed an increased closure of the wound and hair growth in comparison to topical delivery of therapeutics ${ }^{108}$.

As shown, structures like nanotubes, nanopores and nanoparticles can be used in wearables to deliver drugs; however, their size and structure is specific to a drug therefore, they are only limited in administrating a specific drug. Moreover, wearable drug delivery systems based on aforementioned carriers cannot be administered in patients of all age groups as nanotubes and nanoparticles have been found to be difficult to administer to infants and older adults ${ }^{109}$. Ocular wearable drug delivery systems like the smart contact lens for diabetes treatment, have low bioavailability and have limited volume of drug $(\sim 30 \mu \mathrm{L})$ to administer due to the limited precorneal surface area, which decreases the efficacy of the drug ${ }^{110}$. Furthermore, more advancements in drugs are required in developing wearable drug delivery systems with accurate targeted delivery of drug, as many drugs are not been able to cross the blood brain barrier (BBB) ${ }^{111}$. Moreover, the limited biodiversity of drugs limits the effectiveness of the wearable drug delivery systems ${ }^{112}$.

\section{COMMERCIALLY AVAILABLE HWDS}

In recent times, a number of HWDs have been commercially available in the market. Biocompatibility, accuracy, comfortability, 
Table 1. Motion based health and fitness trackers.

\begin{tabular}{lllll}
\hline & Misfit Shine & Fitbit inspire 2 & Withings pulse & Apple watch \\
\hline Charging & 6 months & 10 days & 50 days & $18 \mathrm{~h}$ \\
Weight $(\mathrm{g})$ & 172.9 & 30 & $7.94 \mathrm{~g}$ & $30.5 \mathrm{~g}$ \\
Dimensions & $5.24 \times 1.97 \times 5.91$ & $1.47 \times 0.66 \times 0.51$ & $0.41 \times 0.71 \times 1.75$ & $1.34 \times 1.57 \times 0.41$ \\
Price & $\$ 69.99$ & $\$ 99.95$ & $\$ 99.95$ & $\$ 399$ \\
Water resistant & Swim-proof & Resistant to $50 \mathrm{~m}$ & Resistant to $50 \mathrm{~m}$ & Resistant to $50 \mathrm{~m}$ \\
\hline
\end{tabular}

and uninterrupted battery performance are essential factors for successful commercializarion of HWDs. This section highlights some of the most recent commercially available HWDs for the continuous monitoring of different biomarkers. The major focus of the commercially available HWDs has been on the motion trackers ${ }^{113}$. Human movement assessment plays an important role for the biomechanics and gait analysis and therefore can be used for diagnosing neurological and psychological diseases. Moreover, motion trackers can keep a track of step counts, which helps in maintaining a record of calories burned. Therefore, a number of such devices are available in the market for this purpose and the most widely used are Misfit Shine, Fitbit Inspire 2, Withings and Apple Smart Watch. Misfit, an American based consumer electronics company, has introduced the Misfit Shine that keeps record of steps count, sleep hours, distance covered, and calories burnt. It has a nonchargeable battery that lasts for 6 months and needs replacement every 6 months. Fitbit, an American based fitness company, has introduced a number of fitness wearables ${ }^{114}$. Fitbit Inspire 2 is one of the recent additions for the real-time and continuous monitoring of vital signs like heart rate, activity, and sleep monitoring. It allows 10 days of charging capacity for continuous monitoring ${ }^{113}$. Moreover, Withings Pulse, is a smart fitness tracker by Withings, a French electronics consumer company ${ }^{115}$. Withings Pulse allows $24 / 7$ monitoring of heart rate and also keeps a track of sleep hours. Its battery lasts for 20 days and is water resistant to up to $50 \mathrm{~m}^{113}$. In contrast to aforementioned fitness wearables, Apple watch comes with features like irregular rhythm notifications, ECG application, and blood oxygen application. Irregular rhythms in heart rate may be suggestive of atrial fibrillation, where atrial fibrillation can lead to stroke, heart failure, and other heart related complications ${ }^{116}$. However, Apple watch cannot constantly look for atrial fibrillation and cannot detect all instances of atrial fibrillation. It only sends notification if irregular rhythms are detected repeatedly ${ }^{116}$. The authenticity of the Apple watch for the detection of atrial fibrillation was confirmed by a study conducted on 419,297 participants at Stanford ${ }^{117}$. In this study participants were monitored with Apple watch and on the reciept of a notification for irregular rhythm, were monitored for 90 days for atrial fibrillation using ECG patch. The study diagnosed 34\% participants with atrial fibrillation using the early detection by Apple watch and $84 \%$ of irregular rhythm notifications were found in accordance with the atrial fibrillation ${ }^{117}$. Moreover, the ECG application keeps a track of the irregular rhythm using a built-in electrical heart sensor and provides notification of atrial fibrillation or normal sinus rhythm. Moreover, it also records the level of oxygen in blood, which helps in the continuous monitoring of hypoxemia, and conditions like asthma, and emphysema ${ }^{116,118}$. However, the blood oxygen application is available only in selected regions ${ }^{116}$. Table 1 summarizes the aforementioned health and fitness trackers based on human motion.

As discussed, ECG is a vital sign that plays an important role in diagnosing and preventing cardiovascular diseases. A number of smart wearables have been introduced in the market for ECG monitoring of ECG. ePatch is an FDA approved, commercially available ECG patch. ePatch is a 3 lead ECG sensor that consists of a sensor housed inside an adhesive patch ${ }^{119}$. The patch can store ECG recordings continuously for 72 hours and results can be analyzed on computer software transmitted using a USB cable ${ }^{119}$. However, the recent advancements in ECG monitors are in leadless acquisition of ECG and LifeTouch sensor is a one such HWD $^{19,120}$. It offers continuous measurement of respiration rate, heart rate, and ECG for verification purposes. Moreover, Savvy is another leadless HWD commercially available HWD for the realtime monitoring of ECG ${ }^{121}$. It is POC in nature and consists of a sensor that attaches with the skin with two adhesive electrodes $^{119}$. These two electrodes are used for measuring ECG during measurement mode and for charging the sensor in charging mode. The battery life of Savvy is up to 20 days $^{119}$. The HWD is housed inside a biocompatible and flexible plastic covering for enduring user's movement. The ECG from the Savvy can be visualized in real time on a mobile application (MobECG), which also allows a summary of the ECG that can be stored or shared with the patient's medical provider ${ }^{119}$. Similarly, ZIO XT and SEEQ are two other important commercially available HWDs for the monitoring of ECG ${ }^{119}$. However, unlike Savvy, ZIO XT, and SEEQ cannot be reused and do not have rechargeable batteries. ZIO XT does not report results in real time; however, it stores data for 14 days after which the ECG patch is returned to the company for data analysis. Similarly, SEEQ, does not report data in real time but instead data is transmitted with the company's cloud and the ECG reporting time depends on the processing time by the company. Table 2 shows some of the commercially available HWDs for ECG monitoring.

Considering the growing market of patients with diabetes, a number of HWDs have been introduced for the continuous monitoring of glucose. Such devices are generally known as CGM, continuous glucose monitor ${ }^{122}$. Notable CGMs are Dexcom G6, Abbott's FreeStyle Libre System, and Medtronic Guardian Connect ${ }^{123-125}$. Dexcom G6 consists of a sensor, transmitter, and a receiver ${ }^{122,123}$. With an automatic applicator, the sensor wire is inserted just under the skin of wearer where sensor measures the glucose readings. However, Dexcom G6 takes 2 hours of calibration after insertion. Readings from the G6 CGM are transmitted to the receiver where they can be visualized by the user in real time on the Dexcom mobile application. However, the application is only compatible with selected mobile devices. Dexcom G6 is one of the few CGMs commercially available along with Abbott's FreeStyle Libre System to measure accurate glucose readings if the patient is taking acetaminophen. Acetaminophen is an analgesic medicine that interferes with CGS and causes inaccurate glucose readings ${ }^{122,126}$. Abbott's FreeStyle Libre comes with a sensor and a reader where sensor is a small minimally invasive device to measure glucose from blood and sends data to the reader where the glucose levels can be visualized. Abbott's CGM placement can only work accurately if placed at the back of the upper arm and unlike Dexcom G6 it takes $12 \mathrm{~h}$ to calibrate $^{122,124}$. Similarly, Medtronic Guardian connect is another CGM which like aforementioned CGMs consists of a sensor but does not contain a reader. The sensor takes $12 \mathrm{~h}$ for calibration and can send data directly to a mobile application instead of a reader device. An important feature about Guardian Connect is its 
Table 2. Commercially available ECG HWDs.

\begin{tabular}{lllll}
\hline & ePatch & Savvy & ZIO XT \\
\hline Battery & $72 \mathrm{~h}$ & 20 days & 14 days \\
No. of ECG channels & 3 & 1 & 1 & 1 \\
Real-time analysis & Using computer software & Mobile application with cloud server & No & No \\
Symptom alert & No & Yes & Yes & 34 \\
Weight $(\mathrm{g})$ & 16 & 21 & $123 \times 53 \times 10.7$ & 50 \\
Dimensions $(\mathrm{mm})$ & $40 \times 49 \times 10$ & $130 \times 35 \times 14$ & $160 \times 60 \times 15$ \\
\hline
\end{tabular}

\begin{tabular}{|c|c|c|c|c|}
\hline Daily calibration & Yes & No & Yes & No \\
\hline Cost & $\begin{array}{l}\text { Subscription based with } \$ 299 \\
\text { per month }\end{array}$ & $\begin{array}{l}\$ 10-\$ 75 \text { per month depending on } \\
\text { insurance }\end{array}$ & $\begin{array}{l}\$ 50 \text { per sensor } \\
\text { per month }\end{array}$ & $\$ 9$ including 50 test strips \\
\hline Company & Dexcom & Abbott & Medtronic & ReliOn \\
\hline
\end{tabular}

Sugar $\mathrm{IQ}^{122}$. Sugar IQ helps user in understanding the glucose patterns based on their daily glucose trends along with insulin intake. It is the only CGM to send predictive notifications 10-60 min beforehand. Table 3 below summarizes above mentioned CGMs along with their cost analyses. It can be seen that these CGMs are costly in comparison to the widely used invasive blood-based glucose meters and it is recommended to follow blood-based glucose meters in case CGMs readings do not match with the symptoms.

\section{CHALLENGES AND FUTURE PERSPECTIVES}

HWDs have extensively revamped the field of healthcare. As discussed, they have proved to be efficacious in monitoring many physiological parameters. However, there is still a plenty of room for improvement in these HWDs due to their limitations that include the stability, sensitivity, privacy, power source, and limited applications for psychological diseases.

As most of wearables involve interactions with the epidermis, the data received from the sensors in these HWDs may experience distortion and noise. Reasons for the noise include constant body motion and hairs on the skin that lead to minimum adhesion between the skin and the wearable. It has been observed that HWDs find extensive applications for monitoring purposes; therefore, more efforts are required to make them suitable for diagnostic purposes. This is because most of the diagnostic techniques involve use of samples like blood, urine, and saliva and HWDs find limited integration with these samples. Therefore, more efforts are required for the integration of HWDs with the platforms that can support the use of biological samples with HWDs and can be used by the end user. Moreover, the use of Al algorithms for example, supervised learning regression algorithm can be used for keeping in track of the behavior of multiple parameters for prognosis ${ }^{127}$. Similarly, security of the wearer is important and as HWDs contain protected health information (PHI), an uncompromised privacy is required ${ }^{128,129}$. For this purpose, secure communication protocols in HWDs are essential in order to ensure security and privacy of wearers ${ }^{130}$.

Furthermore, one of the major limitations that hamper the utility of wearable devices, is a continuous power supply ${ }^{131-133}$. Batteries used in wearables have limited space as they are required to align with the design of wearables. To overcome this limitation, efficient energy harvesters with better power management are required. Self-powered sensors like piezoelectric nanogenerator (PENG) and triboelectric nanogenerator (TENG) are miniaturized energy harvesters. These self-powered sensors involve the conversion of one form of energy, for example, mechanical energy into electrical energy and ensure comfort and miniaturization of HWDs ${ }^{134}$. It has also been observed that HWDs find numerous applications for monitoring physiological diseases like cardiovascular diseases, muscle disorders, blood, and glucose levels. However, limited applications of HWDs for the psychological diseases are available for example, PD, AD, and other psychological disorders. Therefore, an increased utilization of HWDs for psychological diseases can prove to be beneficial in near future.

\section{CONCLUSION}

Wearables are widely used in healthcare for monitoring and diagnostic purposes, because of the comfortability and daily care they provide to the wearer. This paper reviews recent efforts in the use of different HWDs for the monitoring of different disease conditions and have also highlighted recent commercially available HWDs. For this purpose, skin-based HWDs, biofluidicbased HWDs, and other competing wearable technologies have been discussed. The relevant techniques for noninvasive and minimally invasive monitoring of different biological and chemical parameters including blood pressure, heart rate, and perspiration rate have been reviewed. Moreover, applications of HWDs for monitoring of different physiological and psychological parameters have also been discussed. Furthermore, this review also highlights the use of different materials for monitoring, diagnosing, and treating of different diseases, as well as the techniques for the application of HWDs as drug delivery systems including transdermal drug delivery systems and touch actuated systems. Inherent limitations with these wearable devices along with their future perspectives have also been discussed.

\section{DATA AVAILABILITY}

The data that support the findings of this study are available within the paper or available from the corresponding author upon reasonable request. Moreover, sources of all the figures are provided with the paper. 
Received: 22 August 2020; Accepted: 16 March 2021; Published online: 12 April 2021

\section{REFERENCES}

1. Amine, E. K. et al. Diet, nutrition and the prevention of chronic diseases. World Heal. Organ. Tech. Rep. Ser. https://doi.org/10.1093/ajcn/60.4.644a (2003).

2. Someya, T., Bao, Z. \& Malliaras, G. G. The rise of plastic bioelectronics. Nature 540, 379-385 (2016).

3. Xie, J., Chen, Q., Shen, H. \& Li, G. Review-Wearable Graphene Devices for Sensing. J. Electrochem. Soc. 167, 037541 (2020).

4. Kozitsina, A. N. et al. Sensors based on bio and biomimetic receptors in medical diagnostic, environment, and food analysis. Biosensors 8, 1-34 (2018).

5. Bhalla, N., Jolly, P., Formisano, N. \& Estrela, P. Introduction to biosensors. Essays Biochem. 60, 1-8 (2016).

6. Kim, J., Campbell, A. S., de Ávila, B. E. F. \& Wang, J. Wearable biosensors for healthcare monitoring. Nat. Biotechnol. 37, 389-406 (2019).

7. Salim, A. \& Lim, S. Recent advances in noninvasive flexible and wearable wireless biosensors. Biosens. Bioelectron. 141, 111422 (2019).

8. Stylios, G. K. Novel smart textiles. Materials 13, 10-12 (2020).

9. Mohamadzade, B. et al. Recent advances in fabrication methods for flexible antennas in wearable devices: State of the Art. Sensors 19, 2312 (2019).

10. Seshadri, D. R. et al. Wearable sensors for monitoring the internal and external workload of the athlete. npj Digit. Med. 2, 71 (2019).

11. Min, W. \& Jake, L. Wearable technology applications in healthcare: a literature review. Online J. Nurs. Informatics Contrib. https://www.himss.org/resources/ wearable-technology-applications-healthcare-literature-review (2019).

12. García Núñez, C., Manjakkal, L. \& Dahiya, R. Energy autonomous electronic skin. npj Flex. Electron 3, 1, https://doi.org/10.1038/s41528-018-0045-x1 (2019).

13. Rodgers, M. M., Pai, V. M. \& Conroy, R. S. Recent advances in wearable sensors for health monitoring. IEEE Sens. J. 15, 3119-3126 (2015).

14. Yadav, K. S., Kapse-Mistry, S., Peters, G. J. \& Mayur, Y. C. E-drug delivery: a futuristic approach. Drug Discov. Today 24, 1023-1030 (2019).

15. Ouyang, Q. et al. Self-powered, on-demand transdermal drug delivery system driven by triboelectric nanogenerator. Nano Energy 62, 610-619 (2019).

16. Jayaneththi, V. R. et al. Controlled transdermal drug delivery using a wireless magnetic microneedle patch: Preclinical device development. Sens. Actuators B Chem. 297, 126708 (2019).

17. Massaroni, C. et al. Smart textile for respiratory monitoring and thoracoabdominal motion pattern evaluation. J. Biophotonics 11, 1-12 (2018).

18. Alizadeh Meghrazi, M. et al. Multichannel ECG recording from waist using textile sensors. Biomed. Eng. Online 19, 1-18 (2020).

19. Choudhry, N. A., Rasheed, A., Ahmad, S., Arnold, L. \& Wang, L. Design, development and characterization of textile stitch-based piezoresistive sensors for wearable monitoring. IEEE Sens. J. 20, 10485-10494 (2020).

20. Gong, Z. et al. Wearable fiber optic technology based on smart textile: a review. Materials 12, 3311 (2019).

21. Jayathilaka, W. A. D. M. et al. Significance of nanomaterials in wearables: a review on wearable actuators and sensors. Adv. Mater. 31, 1-21 (2019).

22. Modali, A., Vanjari, S. R. K. \& Dendukuri, D. Wearable woven electrochemical biosensor patch for non-invasive diagnostics. Electroanalysis 28, 1276-1282 (2016).

23. Yapici, M. K. \& Alkhidir, T. E. Intelligent medical garments with graphenefunctionalized smart-cloth ECG sensors. Sensors 17, 1-12 (2017).

24. Hajizadegan, M. et al. Graphene sensing modulator: toward low-noise, selfpowered wireless microsensors. IEEE Sens. J. 17, 7239-7247 (2017).

25. Arquilla, K., Webb, A. K. \& Anderson, A. P. Textile electrocardiogram (Ecg) electrodes for wearable health monitoring. Sensors 20, 1-13 (2020).

26. Wicaksono, I. et al. A tailored, electronic textile conformable suit for large-scale spatiotemporal physiological sensing in vivo. npj Flex. Electron 4, 5 (2020).

27. Acar, G. et al. Wearable and flexible textile electrodes for biopotential signal monitoring: A review. Electron 8, 1-25 (2019).

28. Gao, K.-P. et al. Wearable multifunction sensor for the detection of forehead EEG signal and sweat rate on skin simultaneously. IEEE Sens. J. 1748, 1-1 (2020).

29. Clinic, M. Electromyography (EMG). https://www.mayoclinic.org/tests-procedures/ emg/about/pac-20393913 (2021).

30. Pino, E. J., Arias, Y. \& Aqueveque, P. Wearable EMG shirt for upper limb training. Proc. Annu. Int. Conf. IEEE Eng. Med. Biol. Soc. EMBS 2018, 4406-4409 (2018).

31. Dimitrov, G. V. et al. Muscle fatigue during dynamic contractions assessed by new spectral indices. Med. Sci. Sports Exerc. 38, 1971-1979 (2006).

32. Someya, T. \& Amagai, M. Toward a new generation of smart skins. Nat. Biotechnol. 37, 382-388 (2019).

33. Kabiri Ameri, S. et al. Graphene electronic tattoo sensors. ACS Nano 11, 7634-7641 (2017).
34. Park, D. Y. et al. Self-powered real-time arterial pulse monitoring using ultrathin epidermal piezoelectric sensors. Adv. Mater. 29, 1-9 (2017)

35. Measuring Pulse Rate. The arterial pulse is a,contraction and increase in volume. http://healthcaresciencesocw.wayne.edu/vs/4_2.htm\#: :text. (2020).

36. Colin, G. C. et al. Pulmonary hypertension due to left heart disease: diagnostic value of pulmonary artery distensibility. Eur. Radiol. https://doi.org/10.1007/ s00330-020-06959-7 (2020).

37. Schettler, G. Prevention of cardiovascular disease. Lebensversicherungsmedizin. https://www.who.int/cardiovascular_diseases/guidelines/Fulltext.pdf (2007).

38. Photonics, I. LLO Laser Lift Off. https://www.ipgphotonics.com/en/applications/ micromachining/laser-lift-off (2020).

39. World Health Organization, W. Hypertension. https://www.who.int/news-room/ fact-sheets/detail/hypertension (2020).

40. Fletcher, G. B. Blood pressure readings. J. Am. Med. Assoc. 144, 201 (1950).

41. Luo, N. et al. Flexible piezoresistive sensor patch enabling ultralow power cuffless blood pressure measurement. Adv. Funct. Mater. 26, 1178-1187 (2016).

42. Castaneda, D. et al. A review on wearable photoplethysmography sensors and their potential future applications in health care. Int. J. Biosens. Bioelectron 4, 139-148 (2018).

43. Food and Drug Administration Staff. Biocompatibility Testing of Medical Devices. vol. 0889. https://www.fda.gov/regulatory-information/search-fda-guidance-documents/ biocompatibility-testing-medical-devices-standards-specific-informationaccreditation-scheme (2020).

44. Schreiner, C., Catherwood, P., Anderson, J. \& McLaughlin, J. Blood oxygen level measurement with a chest-based Pulse Oximetry prototype System. Comput. Cardiol. 37, 537-540 (2010).

45. Savarese, G. \& Lund, L. H. Pharmacological therapy nitrates as a treatment of acute heart failure pharmacological therapy. J. Hear. Team 3, 51-55 (2016).

46. Dovancescu, S. et al. Detecting heart failure decompensation by measuring transthoracic bioimpedance in the outpatient setting: rationale and design of the SENTINEL-HF study. JMIR Res. Protoc. 4, e121 (2015).

47. Cuba Gyllensten, I. et al. Early indication of decompensated heart failure in patients on home-telemonitoring: a comparison of prediction algorithms based on daily weight and noninvasive transthoracic bio-impedance. JMIR Med. Inform. 4, e3 (2016).

48. Hafid, A. et al. Full impedance cardiography measurement device using raspberry $\mathrm{PI} 3$ and system-on-chip biomedical instrumentation solutions. IEEE J. Biomed. Heal. Inform. 22, 1883-1894 (2018).

49. $\mathrm{Yu}, \mathrm{C}$. M. et al. Intrathoracic impedance monitoring in patients with heart failure: correlation with fluid status and feasibility of early warning preceding hospitalization. Circulation 112, 841-848 (2005).

50. OuraRing. Oura Ring: the most accurate sleep and activity tracker. https:// ouraring.com/ (2020).

51. Yan, Z. Unprecedented pandemic, unprecedented shift, and unprecedented opportunity. Hum. Behav. Emerg. Technol. 2, 110-112 (2020).

52. Gorbalenya, A. E. et al. The species Severe acute respiratory syndrome-related coronavirus: classifying 2019-nCoV and naming it SARS-CoV-2. Nat. Microbiol. 5, 536-544 (2020).

53. Kabir, M. A. et al. Diagnosis for COVID-19: current status and future prospects. Expert. Rev. Mol. Diagn. https://doi.org/10.1080/14737159.2021.1894930 (2021).

54. WHO. WHO Situation reports-Coronavirus disease 2019. https://www.who.int/ emergencies/diseases/novel-coronavirus-2019/ (2020).

55. Etherington, D. Researchers use biometrics, Startups Ring, to including data from the Oura predict COVID-19 symptoms. https://techcrunch.com/2020/05/28/ researchers-use-biometrics-including-data-from-the-oura-ring-to-predict-covid19-symptoms-in-advance/ (2020).

56. Ring, O., Ring, O. \& Ring, O. Oura Ring. https://support.ouraring.com/hc/en-us/ articles/360051642914-How-Long-Does-the-Oura-Ring-Battery-Last-\#: : text $=$ Onaverage $\% 2$ C. (2020).

57. Kim, J. et al. Wearable salivary uric acid mouthguard biosensor with integrated wireless electronics. Biosens. Bioelectron 74, 1061-1068 (2015).

58. Mannoor, M. S. et al. Graphene-based wireless bacteria detection on tooth enamel. Nat. Commun. 3, 763 (2012).

59. Lee, C., Wei, X., Kysar, J. W. \& Hone, J. Measurement of the Elastic Properties and Intrinsic Strength of Monolayer Graphene. Science (80-.). 321, 385-388 (2008).

60. World Health Organization. E. coli. https://www.who.int/news-room/fact-sheets/ detail/e-coli (2020).

61. Centers for Disease Control. E. coli (Escherichia coli). https://www.cdc.gov/ecoli/ index.html (2020).

62. Dixon, M. F. Patterns of inflammation linked to ulcer disease. Bailliere's Best. Pract. Res. Clin. Gastroenterol. 14, 27-40 (2000).

63. Robert Roger Lebel, S. J. Letters to the editors. Med. Biol. Eng. 11, 516 (1973).

64. Chen, Y. et al. Flexible inorganic bioelectronics. npj Flex. Electron. 4, 1-20 (2020). 
65. Pierleoni, P. et al. A smart inertial system for $24 \mathrm{~h}$ monitoring and classification of tremor and freezing of gait in Parkinson's disease. IEEE Sens. J. 19, 11612-11623 (2019).

66. Talitckii, A. et al. Avoiding misdiagnosis of Parkinson's disease with the use of wearable sensors and artificial intelligence. IEEE Sens. J. XX, 1-1 (2020).

67. Samii, A., Nutt, J. G. \& Ransom, B. R. Parkinson's disease Alzheimer's disease. Lancet 363, 2-6 (2004).

68. Mhyre, T. R., Boyd, J. T., Hamill, R. W. \& Maguire-Zeiss, K. A. Parkinson's Disease. vol. 65 (Subcellular Biochemistry, 2012).

69. Lebouvier, T. et al. The second brain and Parkinson's disease. Eur. J. Neurosci. 30, 735-741 (2009).

70. Silva de Lima, A. L. et al. Home-based monitoring of falls using wearable sensors in Parkinson's disease. Mov. Disord. 35, 109-115 (2020).

71. Lonini, L. et al. Wearable sensors for Parkinson's disease: which data are worth collecting for training symptom detection models. npj Digit. Med. 1, 64 (2018).

72. Slowness, M. \& Levodopa, M. Bradykinesia (slowness of movement). Parkinson's Foundation. https://www.parkinson.org/Understanding-Parkinsons/Symptoms/ Movement-Symptoms/Bradykinesia-Slowness-of-Movement (2020).

73. American Parkinson Disease Association. Common Symptoms Of Parkinson's Disease. 1-7. https://www.apdaparkinson.org/what-is-parkinsons/symptoms/ (2020).

74. WHO. Fact sheets. Dementia 19. Https://Www.Who.Int/News-Room/Fact-Sheets/ Detail/Dementia (2020).

75. World Health Organization. 10 facts on dementia. https://www.who.int/features/ factfiles/dementia/en/ (2020).

76. Barry, P., Dockery, J., Littman, D. \& Barry, M. Intelligent assistive technologies. Presence Teleoperators Virtual Environ. 3, 208-215 (1994)

77. Lai Kwan, C. et al. Wearable technology for detecting significant moments in individuals with dementia. Biomed Res. Int. 2019, 1-13 (2019).

78. Lee, J. H. et al. Flexible conductive composite integrated with personal earphone for wireless, real-time monitoring of electrophysiological signs. ACS Appl. Mater. Interfaces 10, 21184-21190 (2018).

79. Iqbal, S. M. A. \& Butt, N. Z. Design and analysis of microfluidic cell counter using spice simulation. arXiv 1, 1-10 (2019).

80. Padash, M., Enz, C. \& Carrara, S. Microfluidics by additive manufacturing for wearable biosensors: a review. Sensors 20, 1-28 (2020).

81. Li, S., Ma, Z., Cao, Z., Pan, L. \& Shi, Y. Advanced wearable microfluidic sensors for healthcare monitoring. Small 16, 1903822 (2020).

82. Thomas, S. Research highlights. Nat. Electron. https://doi.org/10.1039/c1lc90074a (2020).

83. Koh, A. et al. A soft, wearable microfluidic device for the capture, storage, and colorimetric sensing of sweat. Sci. Transl. Med. 8, 366ra165-366ra165 (2016).

84. Green, A. \& Kirk, J. Guidelines for the performance of the sweat test for the diagnosis of cystic fibrosis. Ann. Clin. Biochem. 44, 25-34 (2007).

85. FitzSimmons, S. C. The changing epidemiology of cystic fibrosis. J. Pediatr. 122 1-9 (1993).

86. Coleman, B., Coarsey, C., Kabir, M. A. \& Asghar, W. Point-of-care colorimetric analysis through smartphone video. Sens. Actuators B Chem. 282, 225-231 (2019).

87. Kabir, M. A., Zilouchian, H., Sher, M. \& Asghar, W. Development of a flow-free automated colorimetric detection assay integrated with smartphone for Zika NS1. Diagnostics 10, 1-11 (2020).

88. Gonzalez-Fernandez, T., Tenorio, A. J., Kent Leach, J. \& Kent Leach, J. Threedimensional printed stamps for the fabrication of patterned microwells and high-throughput production of homogeneous cell spheroids. 3D Print. Addit. Manuf. 7, 139-147 (2020).

89. Kamei, K. I. et al. 3D printing of soft lithography mold for rapid production of polydimethylsiloxane-based microfluidic devices for cell stimulation with concentration gradients. Biomed. Microdevices 17, 1-8 (2015).

90. Choi, J., Kang, D., Han, S., Kim, S. B. \& Rogers, J. A. Thin, soft, skin-mounted microfluidic networks with capillary bursting valves for chrono-sampling of sweat. Adv. Healthc. Mater. 6, 1-10 (2017).

91. Anastasova, S. et al. A wearable multisensing patch for continuous sweat monitoring. Biosens. Bioelectron. 93, 139-145 (2017).

92. Ardalan, S., Hosseinifard, M., Vosough, M. \& Golmohammadi, H. Towards smart personalized perspiration analysis: An loT-integrated cellulose-based microfluidic wearable patch for smartphone fluorimetric multi-sensing of sweat biomarkers. Biosens. Bioelectron. 168, 112450 (2020).

93. WHO. Diabetes. https://www.who.int/es/news-room/fact-sheets/detail/diabetes (2018).

94. Saeedi, P. et al. Global and regional diabetes prevalence estimates for 2019 and projections for 2030 and 2045: Results from the International Diabetes Federation Diabetes Atlas, 9th edition. Diabetes Res. Clin. Pract. 157, 107843 (2019).

95. Sen, D. K. \& Sarin, G. S. Tear glucose levels in normal people and in diabetic patients. Br. J. Ophthalmol. 64, 693-695 (1980).
96. Duffy, M. The FDA Approves Marketing of the Triggerfish; Contact Lens Sensor to Monitor Glaucoma Eye Pressure. VisionAware 1-5. https://www.visionaware. org/blog/visionaware-blog/the-fda-approves-marketing-of-the-triggerfishsmart-contact-lens-sensor-to-monitor-glaucoma-eye-pressure-2338/12 (2016).

97. Keum, D. H. et al. Wireless smart contact lens for diabetic diagnosis and therapy. Sci. Adv. 6, eaba3252 (2020).

98. Lin, Y. R. et al. Noninvasive glucose monitoring with a contact lens and smartphone. Sensors 18, 1-12 (2018).

99. Halldorsson, S., Lucumi, E., Gómez-Sjöberg, R. \& Fleming, R. M. T. Advantages and challenges of microfluidic cell culture in polydimethylsiloxane devices. Biosens. Bioelectron. 63, 218-231 (2015).

100. Chiu, D. T. et al. Small but perfectly formed? Successes, challenges, and opportunities for microfluidics in the chemical and biological sciences. Chem. 2, 201-223 (2017).

101. Brandt, J. D. et al. Long-term safety and efficacy of a sustained-release bimatoprost ocular ring. Ophthalmology 124, 1565-1566 (2017).

102. $\mathrm{Wu}, \mathrm{C}$. et al. Self-powered iontophoretic transdermal drug delivery system driven and regulated by biomechanical motions. Adv. Funct. Mater. 30, 1-7 (2020).

103. Shouchane-Blum, K., Geffen, N. \& Zahavi, A. Sustained drug delivery platformsā new era for glaucoma treatment. Clin. Exp. Vis. Eye Res. 2, 22-29 (2019).

104. Shahbazi, M.-A. et al. A self-healable, moldable and bioactive biomaterial gum for personalised and wearable drug delivery. J. Mater. Chem. B https://doi.org/ 10.1039/C9TB02156F (2020).

105. Kim, B. et al. Touch-actuated transdermal delivery patch for quantitative skin permeation control. Sens. Actuators B Chem. 256, 18-26 (2018).

106. $\mathrm{Di}, \mathrm{J}$. et al. Stretch-triggered drug delivery from wearable elastomer films containing therapeutic depots. ACS Nano 9, 9407-9415 (2015).

107. Ly, T. T., Layne, J. E., Huyett, L. M., Nazzaro, D. \& O'Connor, J. B. Novel bluetoothenabled tubeless insulin pump: innovating pump therapy for patients in the digital age. J. Diabetes Sci. Technol. 13, 20-26 (2019).

108. Derakhshandeh, $\mathrm{H}$. et al. A wirelessly controlled smart bandage with 3D-printed miniaturized needle arrays. Adv. Funct. Mater. 30, 1-11 (2020).

109. Bhagwat, R. R. \& Vaidhya, I. S. Novel drug delivery systems: An Overview. Int. J. Pharm. Sci. Res. 4, 970-982 (2013).

110. Jumelle, C., Gholizadeh, S., Annabi, N. \& Dana, R. Advances and limitations of drug delivery systems formulated as eye drops. J. Control. Release 321, 1-22 (2020).

111. Dong, X. Current strategies for brain drug delivery. Theranostics 8, 1481-1493 (2018).

112. Singh, A. P., Biswas, A., Shukla, A. \& Maiti, P. Targeted therapy in chronic diseases using nanomaterial-based drug delivery vehicles. Signal Transduct. Target Ther. 4, 1-21 (2019).

113. Haghi, M., Thurow, K. \& Stoll, R. Wearable devices in medical internet of things: scientific research and commercially available devices. Healthc. Inform. Res. 23, 4-15 (2017).

114. Fitbit. Fitbit Inspire 2. 1-7. https://www.fitbit.com/global/us/products/trackers/ inspire2 (2021).

115. Withings. Withings-Pulse HR. Ifri. https://www.withings.com/us/en/pulse-hr (2020).

116. Apple Inc. Apple Watch. Helping your patients identify early warning signs. https:// www.apple.com/healthcare/apple-watch/ (2020).

117. Perez, M. V. et al. Large-scale assessment of a smartwatch to identify atrial fibrillation. N. Engl. J. Med. 381, 1909-1917 (2019).

118. Clinic, M. Symptoms and causes of Emphysema. https://www.mayoclinic.org/ diseases-conditions/emphysema/symptoms-causes/syc-20355555? $\mathrm{p}=1$ (2020).

119. Trobec, R., Tomašić, I., Rashkovska, A., Depolli, M. \& Avbelj, V. Commercial ECG systems. SpringerBriefs Appl. Sci. Technol. https://doi.org/10.1007/978-3-31959340-1_6 (2018).

120. Isansys. Lifetouch. https://www.isansys.com/en/Wearable-Sensors (2020).

121. Savvy. SAVVY ECG Operation c. http://www.savvy.si/en/Savvy_1/ (2020).

122. Funtanilla, V. D., Caliendo, T. \& Hilas, O. Continuous glucose monitoring: a review of available systems. P T 44, 550-553 (2019).

123. Dexcom. The Dexcom G6 CGM System. https://www.dexcom.com/g6-cgmsystem (2020)

124. Abbott. Revolutionizing CGM with Freestyle Libre. https://www.abbott.com/lifechanging-tech/revolutionizing-cgm-with-freestyle-libre.html (2019).

125. Medtronic. The Guardian Connect System. https://www.medtronicdiabetes.com/ products/guardian-connect-continuous-glucose-monitoring-system (2020).

126. Gerriets, V., Anderson, J. \& Nappe, T. M. Acetaminophen. Mech. Action https:// doi.org/10.1007/978-3-642-46051-7 (1967).

127. Mohanta, B., Das, P. \& Patnaik, S. Healthcare 5.0: A paradigm shift in digita healthcare system using artificial intelligence, IOT and $5 \mathrm{G}$ communication. Proc. 2019 Int. Conf. Appl. Mach. Learn. https://doi.org/10.1109/ICAML48257.2019.00044 (2019).

128. Tawalbeh, L., Muheidat, F., Tawalbeh, M. \& Quwaider, M. IoT privacy and security: challenges and solutions. Appl. Sci. 10, 4102 (2020). 
129. Alharbi, R. \& Almagwashi, H. The privacy requirments for wearable loT devices in healthcare domain. Proc. 2019 Int. Conf. Futur. Internet Things Cloud Work. https://doi.org/10.1109/FiCloudW.2019.00017 (2019).

130. Balas, V. E. A Handbook of Internet of Things in Biomedical and Cyber Physical System (Book). vol. 165 (Springer International Publishing, 2020).

131. Chong, Y. W., Ismail, W., Ko, K. \& Lee, C. Y. Energy harvesting for wearable devices: a review. IEEE Sens. J. 19, 9047-9062 (2019).

132. Homayounfar, S. Z. \& Andrew, T. L. Wearable sensors for monitoring human motion: a review on mechanisms, materials, and challenges. SLAS Technol. 25, 9-24 (2020).

133. Ahmad Tarar, A., Mohammad, U. \& Srivastava, S. K. Wearable skin sensors and their challenges: a review of transdermal, optical, and mechanical sensors. Biosensors 10, 56 (2020).

134. Li, Z., Zheng, Q., Wang, Z. L. \& Li, Z. Nanogenerator-based self-powered sensors for wearable and implantable electronics. Research 2020, 1-25 (2020).

\section{ACKNOWLEDGEMENTS}

We would like to thank Md Alamgir Kabir and Natasha Ramnauth for reviewing this manuscript and providing their invaluable comments and suggestion. We acknowledge research support from NSF CAREER Award 1942487, NIH R15AI127214, and the seed award from I-SENSE Institute, and College of Engineering and Computer Science, Florida Atlantic University, Boca Raton, FL.

\section{AUTHOR CONTRIBUTIONS}

S.M.A.I. conducted the research as a lead author and wrote the manuscript under the supervision of W.A. All authors reviewed the manuscript and provided corrections and comments.

\section{COMPETING INTERESTS}

The authors declare no competing interests.

\section{ADDITIONAL INFORMATION}

Correspondence and requests for materials should be addressed to W.A.

Reprints and permission information is available at http://www.nature.com/ reprints

Publisher's note Springer Nature remains neutral with regard to jurisdictional claims in published maps and institutional affiliations.

(i) Open Access This article is licensed under a Creative Commons Attribution 4.0 International License, which permits use, sharing, adaptation, distribution and reproduction in any medium or format, as long as you give appropriate credit to the original author(s) and the source, provide a link to the Creative Commons license, and indicate if changes were made. The images or other third party material in this article are included in the article's Creative Commons license, unless indicated otherwise in a credit line to the material. If material is not included in the article's Creative Commons license and your intended use is not permitted by statutory regulation or exceeds the permitted use, you will need to obtain permission directly from the copyright holder. To view a copy of this license, visit http://creativecommons. org/licenses/by/4.0/.

(c) The Author(s) 2021 